\title{
Ultrafast Electromagnetic Waves Emitted from Semiconductor
}

\author{
YiMing Zhu and SongLin Zhuang \\ Engineering Research Center of Optical Instrument and System, Ministry of Education, \\ University of Shanghai for Science and Technology
}

China

\section{Introduction}

Semiconductor devices have become indispensable for generating electromagnetic radiation in every day applications. Visible and infrared diode lasers are at the core of information technology, and at the other end of the spectrum, microwave and radio frequency emitters enable wireless communications. But the ultrafast electromagnetic waves, whose frequency locates in terahertz $(\mathrm{THz})$ region $\left(0.3-30 \mathrm{THz} ; 1 \mathrm{THz}=10^{12} \mathrm{~Hz}\right)$, has remained largely underdeveloped, despite the identification of various possible applications. One of the major applications of $\mathrm{THz}$ spectroscopy systems is in material characterization, particularly of lightweight molecules and semiconductors [1] [2]. Furthermore, $\mathrm{THz}$ imaging systems may find important niche applications in security screening and manufacturing quality control [3] - [5]. An important goal is the development of three dimensional (3-D) tomographic T-ray imaging systems. THz systems also have broad applicability in a biomedical context, such as the T-ray biosensor [6]. A simple biosensor has been demonstrated for detecting the glycoprotein avidin after binding with vitamin $\mathrm{H}$ (biotin) [7].

However, progresses in these areas have been hampered by the lack of efficient ultrafast electromagnetic wave / THz wave sources. As shown in Fig. 3.1, transistors and other electronic devices based on electron transport are limited to about $\sim 300 \mathrm{GHz}(\sim 50 \mathrm{GHz}$ being the rough practical limit; devices much above that are extremely inefficient) [8]. On the other hand, the wavelength of semiconductor lasers can be extended down to only $\sim 10$ $\mu \mathrm{m}$ (about $\sim 30 \mathrm{THz}$ ) [9]. Between two technologies, lie the so called terahertz gap, where no semiconductor technology can efficiently convert electrical power into electromagnetic radiation. The lack of a high power, low cost, portable room temperature $\mathrm{THz}$ source is the most significant limitation of modern $\mathrm{THz}$ systems. A number of different mechanisms have been exploited to generate $\mathrm{THz}$ radiation, such as photocarrier acceleration in photoconducting antennas, second order nonlinear effects in electro-optical (EO) crystals and quantum cascade laser. Currently, conversion efficiencies in all of these sources are very low, and consequently, average $\mathrm{THz}$ beam powers tend to be in the nanowatt to microwatt range, whereas the average power of the femtosecond optical source is in the region of $\sim 1$ W. There is still a long way to go before commercial devices based on this principle can be mass-produced. 


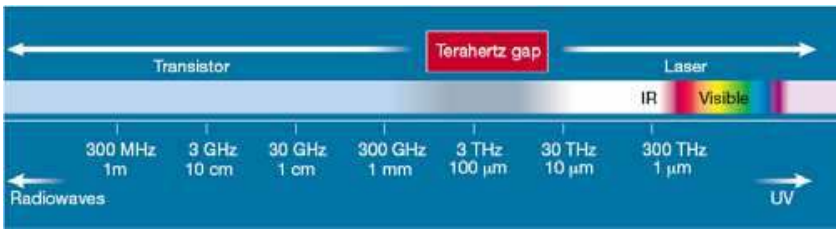

Fig. 3.1. The THz gap. The gap, lying roughly between $300 \mathrm{GHz}$ and $30 \mathrm{THz}$, exists because the frequencies generated by transistors and lasers, typical semiconductor devices, do not overlap. No current semiconductor technology can efficiently convert electrical power into electromagnetism in this range

Photoconduction is one of the most common approaches for generating broadband pulsed ultrafast electromagnetic beams/THz beams. The photoconductive approach uses high speed photoconductors as transient current sources for radiating antennas [10]. Typical photoconductors include high resistivity $\mathrm{GaAs}, \mathrm{InP}$ and radiation damaged silicon wafers. Metallic electrodes are used to bias the photoconductive gap and form an antenna. The physical mechanism for $\mathrm{THz}$ beam generation in photoconductive antennas begins with an ultrafast laser pulse (with a photon energy larger than the bandgap of the material $h v \geq E_{g}$ ), which creates electron and hole pairs in the photoconductor. The free carriers are then accelerated by the static bias field and form a transient photocurrent, and the fast, time varying current radiates electromagnetic waves. The $\mathrm{THz}$ radiation $\mathrm{E}_{\mathrm{THz}}$, emitted from the samples should be proportional to the change of the photocurrent $J$ :

$$
E_{\mathrm{THz}} \propto \frac{\partial J}{\partial t}
$$

Figure 3.2 shows the principle of generating $\mathrm{THz}$ radiation from antenna by photoconduction method. Several material parameters affect the intensity and the bandwidth of the resultant $\mathrm{THz}$ radiation. For efficient $\mathrm{THz}$ radiation, it is desirable to have rapid photocurrent rise and decay time. Thus, semiconductors with small effective electron masses such as InAs and InP are attractive. The maximum drift velocity is also an important material parameter, but it is generally limited by the intraband scattering rate or by intervalley scattering in direct semiconductors such as GaAs [11] [12]. Because the radiating energy mainly comes from stored surface energy in the form of the static bias field, the $\mathrm{THz}$ radiation energy scales up with the bias and optical fluency. The breakdown field of the material is another important parameter because this determines the maximum bias that may be applied. Photoconductive emitters are capable of relatively large average $\mathrm{THz}$ powers in excess of $\sim 40 \mu \mathrm{W}$ [13] and bandwidths as high as $\sim 4 \mathrm{THz}$ [14].

As described above, impulsive currents in semiconductors excited by femtosecond optical pulses radiate ultrafast electromagnetic waves in the $\mathrm{THz}$ frequency range, serving, for example, as an ultrafast electromagnetic wave source for $\mathrm{THz}$ spectroscopy. As described in Eq. (3.1), the ultrafast electromagnetic wave in the far field is proportional to the time derivative of the current, $\partial J / \partial t$. In a simplified view, since $J$ is proportional to carrier velocity which can be written as $J=n e v$, where, $e, n$, and $v$ are the elementary electric charge, total number of photoexcited carriers and there velocity, respectively, $\mathrm{E}_{\mathrm{THz}}$ is proportional to carrier acceleration by assuming that the carriers are excited by sufficiently short laser pulses and their lifetime is long enough ( $n$ is regarded as being constant). Hence, in principle, the observation of such $\mathrm{THz}$ electromagnetic waves provides us with a way of 
looking into the temporal variation of $v$, namely the acceleration / deceleration dynamics of photoexcited carriers [11] [12] [15] [16]. Figure 3.3 shows the illustration of ultrafast electromagnetic waves, $\mathrm{THz}$ waves, emitted from intrinsic bulk GaAs photoexcited by femtosecond laser pulses under bias electric fields. By understanding the nonequilibrium transport of carriers in bulk GaAs, the gain region in the $\mathrm{THz}$ range, can be clarified, which is the necessary condition for the microwave oscillation [17] [18].
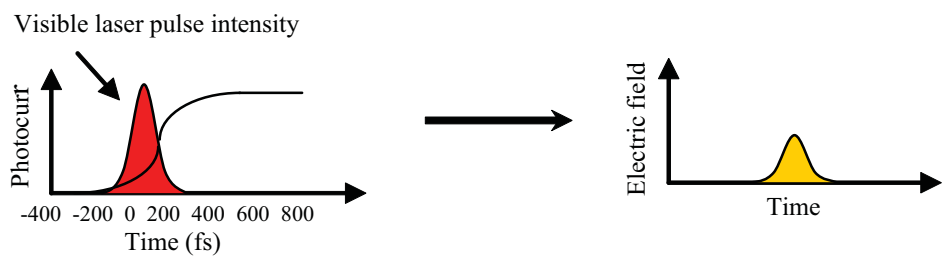

Ti/Au electrodes
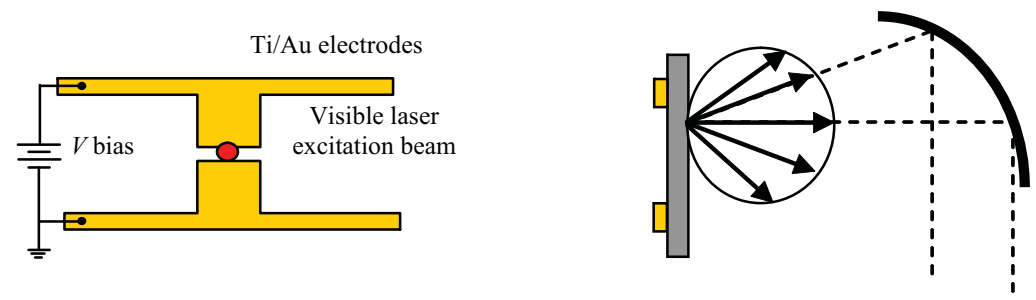

Fig. 3.2. The principle of generating $\mathrm{THz}$ radiation from antenna by photoconduction method

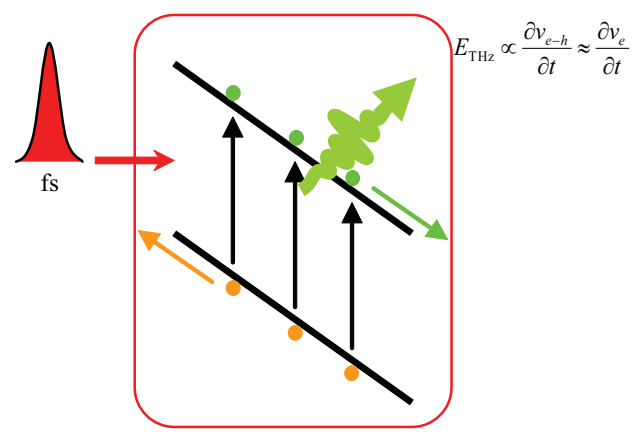

Fig. 3.3. The illustration of generating THz waveforms from intrinsic bulk GaAs photoexcited by femtosecond laser pulses under bias electric fields

The novel experimental technique of the time domain $\mathrm{THz}$ spectroscopy for observing ultrafast carrier motion in the femtosecond time regime together with electromagnetic wave radiation that is proportional to $\mathrm{d} v / \mathrm{d} t$, is introduced in section 2. Invaluable information, femtosecond acceleration of carriers in bulk GaAs under very high electric fields investigated by time domain $\mathrm{THz}$ spectroscopy, contributing to better understanding of device physics in existing high speed electron devices, is discussed in section 3. Finally, the present insights on the gain in GaAs due to electrons intervalley transfer under high electric fields, which is of practical importance for its exploitation in ultrafast electromagnetic wave oscillators, are discussed in section 4. 


\section{Time domain terahertz spectroscopy by electro-optic sampling method}

Time domain terahertz $(\mathrm{THz})$ spectroscopy affords a powerful technique for the research of semiconductors of current industrial interest [2]; the time domain studies of the emitted ultrafast wave / THz wave from various semiconductor structures provide a unique way of looking directly into the temporal as well as spatial evolution of the excited carriers on the sub-picosecond time scale. In this section, time domain THz electro-optic (EO) sampling via the Pockels effect is discussed in detail, including the EO effect, the response spectrum of the EO crystal, and the experimental setup for EO sampling.

Recently, the technique called time domain $\mathrm{THz}$ spectroscopy has been attracting wide interest. Time domain $\mathrm{THz}$ spectroscopy uses short pulses of broadband $\mathrm{THz}$ radiation, which are typically generated using ultrafast laser pulses. This technique has been developed from a work in 1980's at AT\&T Bell Labs and IBM T. J. Watson Research Center [19] [20]. Historically, freely propagating $\mathrm{THz}$ pulses were measured by means of either photoconductive antennas [21] [22] or far infrared interferometic techniques using incoherent detectors such as bolometers [23] [24]. Although the photoconductive antennas have excellent sensitivity, their frequency response is limited by resonant behavior of the Hertzian dipole structure. For the interferometric techniques, the sensitivity is far worse than that of the photoconductive antennas, because the measurement is coherent and its sensitivity is ultimately limited by the thermal background. In addition, bolometers usually require liquid helium cooling.

On the other hand, ultrafast EO sampling has been widely used in the measurement of local transient electric fields in materials [25] [26]. There existed a need to extend the local electric fields measurement to free space. In 1995, three groups reported their first results using free space EO sampling independently, nearly at the same time [27]-[29]. Although the preliminary results were very poor, rapid progress has been made in the intervening years. It turns out that free space EO sampling is a powerful tool for $\mathrm{THz}$ pulse measurement, providing many advantages, such as high sensitivity, ultrabroad frequency response, ease of use, and parallel measurement capability [30]-[35].

Ultrafast carrier motion in the femtosecond time regime accompanies electromagnetic wave radiation, the electric field component of which is proportional to $\mathrm{d} v / \mathrm{d} t$. Therefore, the investigation of such electromagnetic wave (or $\mathrm{THz}$ radiation) allows us a very unique opportunity of looking directly into the acceleration/deceleration dynamics of carriers in semiconductors. In this chapter, time domain $\mathrm{THz}$ spectroscopy based on $\mathrm{EO}$ sampling via the Pockels effect is discussed. Furthermore, the response spectrum of the EO crystals are also discussed and $100 \mu \mathrm{m}$-thick ZnTe, whose response spectrum is flat up to the frequency around $4 \mathrm{THz}$, is chosen as the EO crystal for this experiment.

\subsection{Electro-optic effect}

EO sampling is a phase sensitive detection technique of electromagnetic radiation which measures a birefringence in an EO crystal induced by the incident electromagnetic radiation. This birefringence in the crystal is probed as a phase shift of an optical probe beam. In the time resolved detection of $\mathrm{THz}$ pulse, the transient birefringence is probed with the optical pulses at different time delays.

The principle of EO sampling can be explained as follows. Suppose that the probe beam is propagating in the z-direction and $x, y$ are the crystal axes of the EO crystal as shown in Fig. 3.4. When an electric field is applied to the EO crystal, the electrically induced birefringence 
axes $x^{\prime}$ and $y^{\prime}$ are at an angle of $45^{\circ}$ with respect to $x$ and $y$. If the input beam is polarized along $x$, then the output light can be obtained from the following expression:

$$
\left(\begin{array}{l}
E_{x} \\
E_{y}
\end{array}\right)=\left(\begin{array}{cc}
\cos \left(\frac{\pi}{4}\right) & -\sin \left(\frac{\pi}{4}\right) \\
\sin \left(\frac{\pi}{4}\right) & \cos \left(\frac{\pi}{4}\right)
\end{array}\right)\left(\begin{array}{cc}
\exp (i \Delta) & 0 \\
0 & 1
\end{array}\right)\left(\begin{array}{cc}
\cos \left(\frac{\pi}{4}\right) & \sin \left(\frac{\pi}{4}\right) \\
-\sin \left(\frac{\pi}{4}\right) & \cos \left(\frac{\pi}{4}\right)
\end{array}\right)
$$

where $\Delta=\Gamma_{0}+\Gamma$ is the phase difference between the $x^{\prime}$ and $y^{\prime}$ polarizations, including both the dynamic $(\Gamma, \mathrm{THz}$ induced $)$ and the static $\left(\Gamma_{0}\right.$, from the intrinsic or residual birefringence of the EO crystal and the compensator) phase difference. Following Eq. (3.2), the light intensity in the $x$ and $y$ polarizations is

$$
\left\{\begin{array}{l}
I_{x}=\left|E_{x}\right|^{2}=I_{0} \cos ^{2} \frac{\Gamma_{0}+\Gamma}{2} \\
I_{y}=\left|E_{y}\right|^{2}=I_{0} \sin ^{2} \frac{\Gamma_{0}+\Gamma}{2}
\end{array}\right.
$$

where $I_{0}=E_{0}{ }^{2}$ is the input intensity. It can be seen that $I_{x}$ and $I_{y}$ are complementary, i.e. $I_{x}+I_{y}$ $=I_{0}$. This is the result of energy conservation and follows from the fact that absorption in the crystal has been ignored. To extract the light in the $x$ and $y$ polarizations separately, a Wollaston prism is usually used.

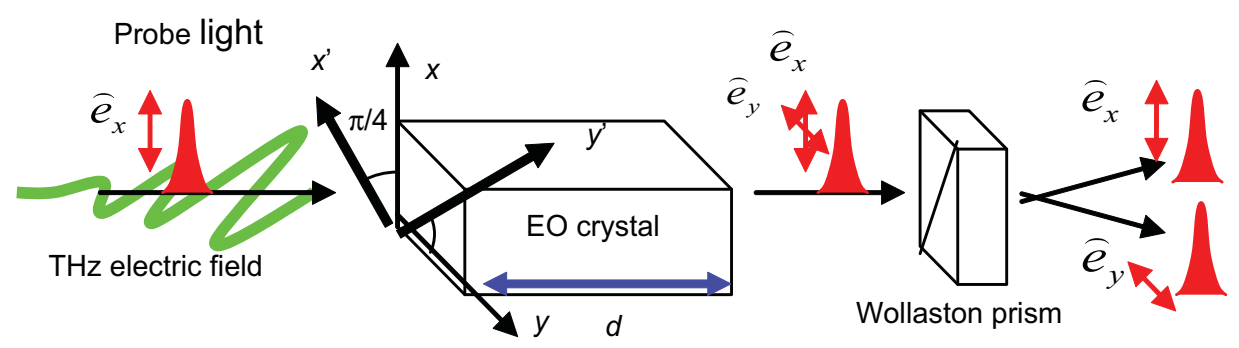

Fig. 3.4. The principle for EO sampling. $x^{\prime}$ and $y^{\prime}$ are the birefringence axes of the EO crystal, $d$ is the thickness of the EO crystal

The static phase term $\Gamma_{0}$ (or called the optical bias) is often set equal to $\pi / 2$ for balanced detection. For an EO crystal without intrinsic birefringence, such as ZnTe, a quarter wave plate is often used to provide this optical bias. Because $|\Gamma|<<1$ in most cases in EO sampling, we have

$$
\left\{\begin{array}{l}
I_{x}=\frac{I_{0}}{2}(1-\Gamma) \\
I_{y}=\frac{I_{0}}{2}(1+\Gamma)
\end{array}\right.
$$


In the two beams, the signals have the same magnitudes but opposite signs. For balanced detection, the difference between $I_{y}$ and $I_{x}$ is measured, giving the signal

$$
I_{s}=I_{y}-I_{x}=I_{0} \Gamma \text {. }
$$

This signal is proportional to the $\mathrm{THz}$ induced phase change, $\Gamma$, and, $\Gamma$, in turn, is proportional to the electric field of the THz pulse, $\mathrm{E}_{\mathrm{THz}}$. For a (110)-oriented ZnTe crystal, the following relation holds:

$$
\Gamma=\frac{\pi n_{g}\left(\lambda_{0}\right)^{3} \gamma_{41} d}{\lambda_{0}} E_{\mathrm{THz}}
$$

where $d$ is the thickness of the crystal, $n_{g}\left(\lambda_{0}\right)$ is the refractive index of the crystal at the wavelength of the optical probe beam $\lambda_{0}$, and $\gamma_{41}$ is the EO coefficient. By detecting the phase retardation $\Gamma$ induced in the $\mathrm{EO}$ crystal by balanced detection as shown in Eq. (3.5), $\mathrm{E}_{\mathrm{THz}}$ can be accurately detected (Eq. (3.6)).

\subsection{The response spectrum of the electro-optic crystal}

For a transient $\mathrm{THz}$ pulse, phase matching should be considered. When the probe pulse has a group velocity different from that of a THz pulse (so-called GVM), the probe does not always sample the same position on the $\mathrm{THz}$ pulse. Instead, it scans across the $\mathrm{THz}$ pulse as the two propagating through the crystal, leading to broadening of the measured $\mathrm{THz}$ waveform. Furthermore, the bandwidth of EO crystal is limited by reststrahlen band of the crystal. To estimate frequency response and upper limit of the EO sensor, we performed a calculation of the theoretical response function of EO crystals, which shows that the importance of properly accounting for the dispersion of the EO coefficient and the GVM.

The complete frequency response function $R(\omega)$ of EO sensors is obtained with the following model: The influences of linear optics such as the GVM between the group velocity of the optical probe pulse and that of the $\mathrm{THz}$ pulse, reflection losses at the detector surface, and absorption inside the crystal have been described recently [36] [37].

After a probe pulse and $\mathrm{THz}$ wave co-propagate through a sensor of thickness $\mathrm{d}$, the accumulated GVM time is

$$
\delta(\omega)=\frac{n_{g}\left(\lambda_{0}\right)-n(\omega)}{c} d,
$$

where $c$ is the speed of the light and $n(\omega)$ is the complex refractive index of the $\mathrm{THz}$ radiation of frequency $\omega$.

Then, the amplitude and its phase of the EO modulation of the optical probe pulse induced by the THz wave is proportional to $d$ as well as the time average of the electric field across the GVM time, $\delta(\omega)$. It has the form as [37],

$$
G(\omega)=\frac{t(\omega)}{\delta(\omega)} \int_{0}^{\delta(\omega)} \exp [i 2 \pi \omega t] d t=t(\omega) \frac{\exp [i 2 \pi \omega \delta(\omega)]-1}{i 2 \pi \omega \delta(\omega)},
$$

where $t(\omega)=2 /[n(\omega)+1]$ is the Fresnel transmission coefficient. 
The group refractive index $n_{g}\left(\lambda_{0}\right)$ at the wavelength of the optical probe pulse is obtained from dispersion data in the near infrared range. The complex refractive index $n(\omega)$ of the $\mathrm{THz}$ radiation can be expressed as:

$$
n(\omega)=\sqrt{\left[1+\left\{\frac{\left(\hbar \omega_{\mathrm{LO}}\right)^{2}-\left(\hbar \omega_{\mathrm{TO}}\right)^{2}}{\left(\hbar \omega_{\mathrm{TO}}\right)^{2}-(\hbar \omega)^{2}-i \hbar \gamma \omega}\right\}\right] \times \varepsilon_{\infty}}
$$

The transverse optical (TO) phonon and longitudinal optical (LO) phonon energies, and the

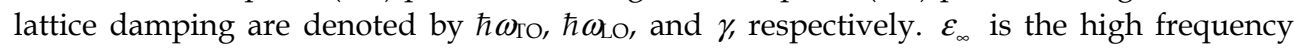
dielectric constant.

$G(\omega)$ describes the detector response if the EO coefficient $\gamma_{41}$ of the sensor material is frequency independent. However, $\gamma_{41}$ exhibits a strong dispersion and resonant enhancement due to the lattice resonance. This effect accounts for very pronounced features in the response functions. An analytic expression for $\gamma_{41}(\omega)$ has been derived in [38], Eq. (5):

$$
\gamma_{41}(\omega)=r_{e} \times\left[1+C\left(1-\frac{(\hbar \omega)^{2}+i \hbar \omega \gamma}{\left(\hbar \omega_{\mathrm{TO}}\right)^{2}}\right)^{-1}\right]
$$

The Faust-Henry coefficient $C$ represents the ratio between the ionic and the electronic part of the EO effect at $\omega=0$. The purely electronic nonlinearity $r_{e}$ is assumed to be constant at the mid and far infrared frequencies $\omega$.

The full complex response function $R(\omega)$ of EO sensor is, then, given by

$$
R(\omega)=G(\omega) \times \gamma_{41}(\omega) .
$$

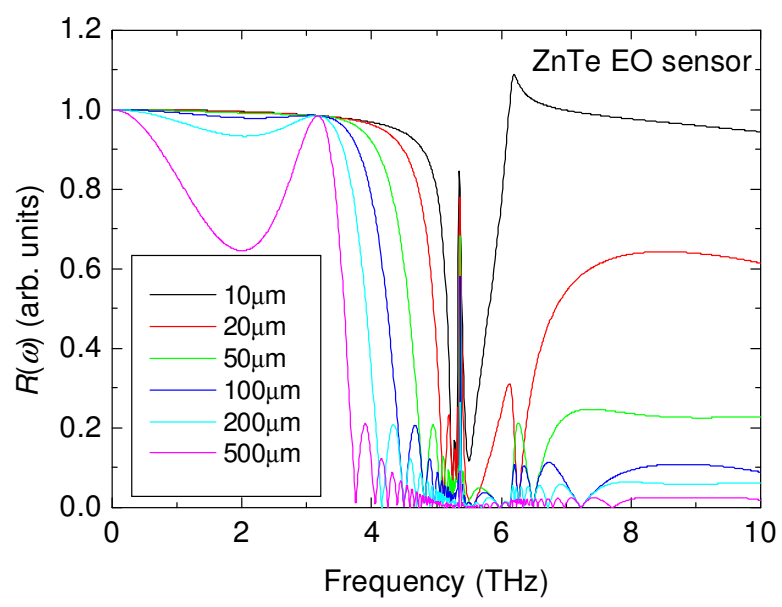

Fig. 3.5. The calculated amplitude response $R(\omega)$ of the ZnTe EO sensor with different thickness, normalized to unity at low frequencies

Figure 3.5 displays the calculated spectra of $|R(\omega)|$ of ZnTe sensors with various thickness, normalized to unity at low frequencies. As shown in Fig. 3.5, a structured dip in the spectra 
appears in the reststrahl region around 5.3 THz. This feature reflects the high absorption of the material and the large velocity mismatch near the lattice resonance. At the TO phonon frequency $(6.2 \mathrm{THz}$ in $\mathrm{ZnTe})$, the nonlinear coefficient $\gamma_{41}(\omega)$ is resonantly enhanced. We can clearly see, with decreasing the thickness of ZnTe crystal, the upper limit of the frequency increases. However, the sensitivity of the EO sensor is proportional to the thickness of the crystal, as shown in Eq. (3.6) in section 2.1. Therefore, a trade-off exists between broadband response and high sensitivity.

\subsection{Time domain terahertz spectroscopy system}

Figure 3.6 shows the setup for free space $\mathrm{THz}$ EO sampling measurements. The ulrafast laser pulse is split by a beam splitter into two beams: a pump beam (strong) and a probe beam (weak). The pump beam illuminates the sample and generates the $\mathrm{THz}$ radiation. The generated $\mathrm{THz}$ radiation is a short electromagnetic pulse with a duration on the order of one picosecond, so the frequency is in the $\mathrm{THz}$ range. The $\mathrm{THz}$ beam is focused by a pair of parabolic mirrors onto an EO crystal. The beam transiently modifies the index ellipsoid of the EO crystal via the Pockels effect, as discussed in detail in section 2.1. The linearly polarized probe beam co-propagates inside the crystal with $\mathrm{THz}$ beam and its phase is modulated by the refractive index change induced by the $\mathrm{THz}$ electric field, $\mathrm{E}_{\mathrm{THz}}$. This phase change is converted to an intensity change by a polarization analyzer (Wollaston prism). A pair of balanced detectors is used to suppress the common laser noise. This also doubles the measured signal. A mechanical delay line is used to change the time delay between the $\mathrm{THz}$ pulse and the probe pulse. The waveform of $\mathrm{E}_{\mathrm{THz}}$ can be obtained by scanning this time delay and performing a repetitive sampling measurement. The principle of $\mathrm{THz}$ signal sampled by the femtosecond pulse is shown in Fig. 3.7. By this sampling method, we do not need the instruments for high speed measurement because the $\mathrm{THz}$ signals are converted to the electrical signals after the sampling by the femtosecond probe pulse. To increase the sensitivity, the pump beam is modulated by a mechanical chopper and $\mathrm{E}_{\mathrm{THz}}$ induced modulation of the probe beam extracted by a lock-in amplifier.

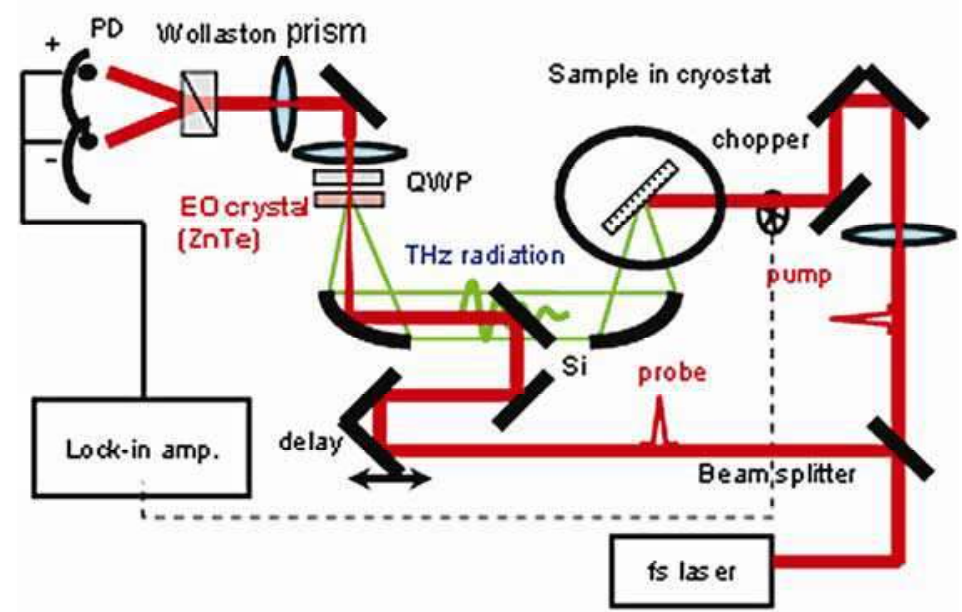

Fig. 3.6. Experimental setup for free space EO sampling 


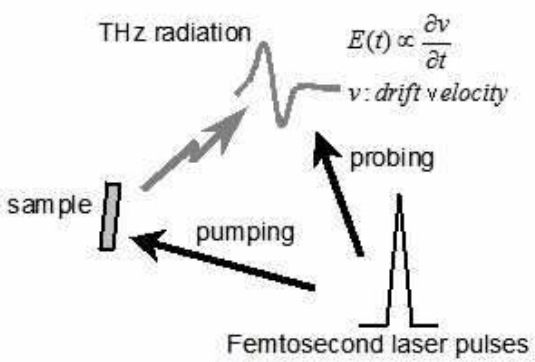

(a)

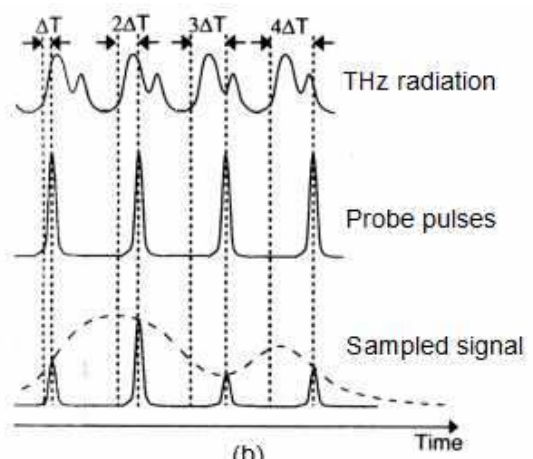

(b)

Fig. 3.7. The principle of $\mathrm{THz}$ signals sampled by the femtosecond probe beam

\section{Femtosecond acceleration of carriers in bulk GaAs}

Ultrafast nonequilibrium transport of carriers in semiconductors biased at high electric fields is of fundamental interest in semiconductor physics. From a fundamental point of view, the detailed understanding of the femtosecond dynamics of carriers in an electric field is a key issue in many body physics. As an example, one may ask whether the welldeveloped semiclassical picture of electronic conduction breaks down on ultrafast time scales and at very high electric fields [39]. Furthermore, clarifying carrier dynamics under extremely nonequilibrium conditions is also strongly motivated by the need to obtain information relevant for the design of ultrahigh speed devices. Indeed, transit times even less than 1 ps have been reported for compound semiconductor field effect transistors [40]. In such ultrashort channel transistors, carriers experience very few scattering events in the channel and drift in a very nonstationary manner as schematically illustrated in the Fig. 3.8. Consequently, the performance of such ultrafast transistors is not mainly determined by the steady-state properties, such as saturation velocities and nobilities, but is governed by the nonstationary carrier transport subjected to high electric fields. It is, therefore, essential to understand nonequilibrium transport of carriers subjected to high electric fields in such devices. However, it has been difficult to characterize such very fast phenomena by using conventional electronics, such as sampling oscilloscopes, because of their limited bandwidth. Consequently, Monte Carlo calculations have been the only tool for discussing transient carrier transport.

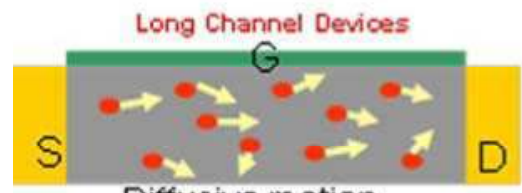

Diffusive motion

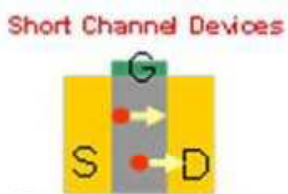

Ballistic motion

Fig. 3.8. Carrier transport in (a) long diffusive channels (b) very short ballistic channels. (a) In conventional long-channel transistors, their performance is determined by steady-state properties, such as saturation velocities and nobilities. However, (b) in very short channel transistors, carrier transport is quasi-ballistic and their switching speed is governed by how fast carriers are accelerated 
So far, only a few experimental studies have been done on the femtosecond carrier acceleration under nonequilibrium in compound semiconductor [11] [12] [15]. Leitenstorfer, et al. presented the first experimental results on the ultrafast electromagnetic wave / terahertz wave emission from electrons and ions in $\mathrm{GaAs}$ and $\mathrm{InP}$ accelerated by very high electric fields and showed firm experimental evidence of velocity overshoot of electrons in the femtosecond time range [11] [12]. They separated the contributions of electrons and lattices, and determine the transient acceleration and velocity of carriers with a time resolution in the order of $10 \mathrm{fs}$. The maximum velocity overshoots and traveling distances during the nonequilibrium regime have been determined for the first time.

In this section, we have investigated nonequilibrium acceleration of carriers in bulk GaAs subjected to very high electric fields by time domain $\mathrm{THz}$ spectroscopy [16]. It is found that $\mathrm{THz}$ emission waveforms have a bipolar feature; i.e., an initial positive peak and a subsequent negative dip. This feature arises from the velocity overshoot. The initial positive peak has been interpreted as electron acceleration in the bottom of $\Gamma$ the valley in GaAs, where electrons have a light effective mass, while the subsequent negative dip has been attributed to intervalley transfer from the $\Gamma$ valley to the $X$ and $L$ valleys.

\subsection{GaAs $m$-i-n diode \& short channel GaAs $m$-i-n diode}

Undoped bulk GaAs sample grown by molecular beam epitaxy was used. Sample \#1 had an $m-i-n$ geometry with a $1 \mu$ m-thick intrinsic GaAs layer. An ohmic contact was fabricated by depositing and annealing AuGeNi alloy on the back side of the sample. A semitransparent NiCr Schottky film was deposited on the surface to apply a DC electric field, $F$, to the intrinsic GaAs layer. The sample was mounted on a copper plate, which was put in the cryostat, for the time domain $\mathrm{THz}$ measurements. The illuminated surface area (window area) was approximately $1.5 \times 1 \mathrm{~mm}^{2}$. The special design of the window size was adopted to avoid the field screening effect.

\subsection{Femtosecond acceleration of carriers in GaAs $m$-i-n diode \& short channel GaAs $m-i-n$ diode}

Femtosecond laser pulses from a mode locked Ti: sapphire laser operated at a repetition rate of $76 \mathrm{MHz}$ was used for time domain $\mathrm{THz}$ spectroscopy. The full width at half maximum (FWHM) of spectral bandwidth of the femtosecond laser pulses was approximately $20 \mathrm{meV}$. The central photon energies of the light pulses were set to $1.422 \mathrm{eV}$ at $300 \mathrm{~K}$ and $1.515 \mathrm{eV}$ at $10 \mathrm{~K}$, in such a way that electrons were created near the bottom of the conduction band, as well as holes near the top of the valence band. The free space EO sampling technique was used to record temporal waveforms of $\mathrm{THz}$ electric fields emitted from the samples [41] [42], as described in chapter 2. The EO sensor used in this experiment was a $100 \square \mu \mathrm{m}$-thick (110)oriented ZnTe crystal. The spectral bandwidth of this sensor was approximately $4 \mathrm{THz}$ [11] [37], as shown in Fig. 3.5 in section 2.2. The corresponding resolution is $\sim 250 \mathrm{fs}$.

Figures 3.9 show temporal waveforms of the THz electric fields emitted from samples \#1 (an $m$-i- $n$ diode with a $1 \mu$ m-thick intrinsic GaAs layer) [Figs. 3.9 (a), 3.9 (b)] for various $F$ at 300 $\mathrm{K}$ and $10 \mathrm{~K}$, respectively. In [43], the position of $t=0$ was empirically determined by choosing a position which does not cause artificial jumps in the phase of Fourier spectra of time domain $\mathrm{THz}$ data. To determine the position of $t=0$ more accurately, we adopted a newly developed method, i.e., the maximum entropy method (MEM) [44]-[46]. The position of $t=0$ set in Figs. 3.9 has been determined by MEM within an error of $\pm 30 \mathrm{fs}$, which is limited by the time interval of the recorded points. 


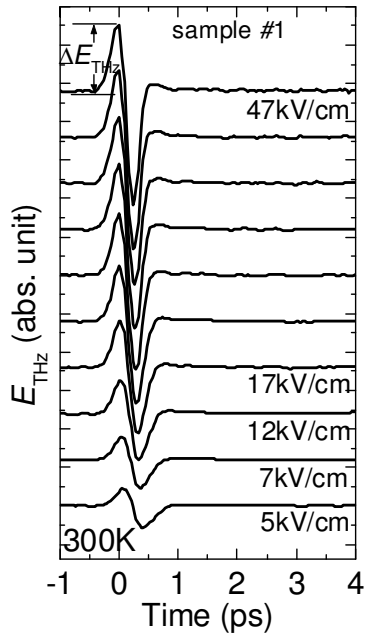

(a)

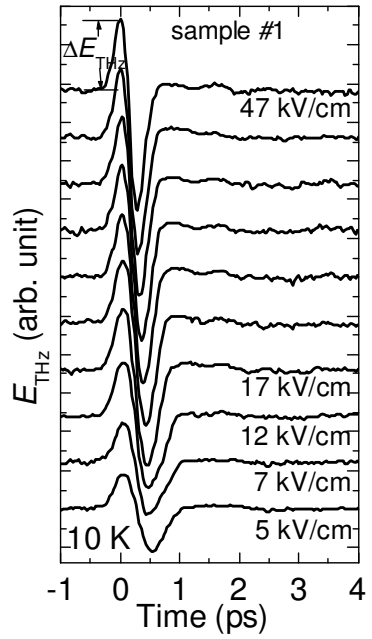

(b)

Fig. 3.9. Bias field dependence of the temporal waveforms of $\mathrm{THz}$ electric field $\left(\mathrm{E}_{\mathrm{THz}}\right)$ emitted from (a) an $m$-i- $n$ diode with $1 \mu \mathrm{m}$-thick intrinsic GaAs layer at $300 \mathrm{~K}$; (b) an $m-i-n$ diode with $1 \mu \mathrm{m}$-thick intrinsic GaAs layer at $10 \mathrm{~K}$;

From the Maxwell equations,

$$
\left\{\begin{array}{l}
\nabla \times E=-\mu_{0} \frac{\partial H}{\partial t} \\
\nabla \times H=J+\frac{\partial D}{\partial t}
\end{array}\right.
$$

THz electric field, $E_{T H z}$, induced by transient current, J, due to photoexcited carriers is given by

$$
E_{\mathrm{THz}} \propto \frac{\partial v}{\partial t}
$$

$E_{T H z}$ is the emitted ultrafast electromagnetic wave, which is proportional to carrier acceleration. The interband transitions induced by the ultrashort excitation pulses create electrons close to the bottom of the $\Gamma$ valley in the conduction band as well as holes near the top of the valence band. In an ideal case without scattering, these electrons are ballistically accelerated by the applied electric field in the $\Gamma$ valley. When electrons in the central valley have gained energy from the applied electric field comparable to the energetic distance between the bottoms of the $\Gamma$ and the satellite valleys, the electrons are scattered to the satellite valleys by longitudinal optical (LO) phonon. Because the effective mass of electrons in the satellite valley is much heavier than that in the central valley, a sudden decrease of drift velocity is expected. Figure 3.10 (b) shows the ideal acceleration of electrons in bulk GaAs when the electrons are in the band as shown in Fig. 3.10 (a). The initial positive part shown in Fig. 3.10 (b) corresponds to ballistic acceleration of electrons in the $\Gamma$ valley and the subsequent sudden dip expresses deceleration due to intervalley transfer from the $\Gamma$ valley to the satellite valleys. 
As shown in Fig. 3.9, the leading edge of the $E_{T H z}$ observed in the experiment is due both to the duration of the femtosecond pulses and to the limitation of the spectral bandwidth of the $\mathrm{EO}$ crystal detector. From the experimental data, we can clearly see the $\mathrm{THz}$ emission waveforms have a bipolar feature; i.e., an initial positive peak and a subsequent negative dip. This feature arises from the velocity overshoot [12]. The initial positive peak has been interpreted as electron acceleration in the $\Gamma$ valley, where electrons have a light effective mass, while the subsequent negative dip has been attributed to the electron deceleration due to intervalley transfer from the $\Gamma$ valley to the $X$ and $L$ valleys. From Figs. 3.9 (a) and (b), we can find at $10 \mathrm{~K}$, the duration time of the waveforms is longer than that at $300 \mathrm{~K}$ at any bias electric fields, $F$, for both samples. The longer duration time of the $\mathrm{THz}$ waveforms at $10 \mathrm{~K}$ is due to the lower scattering rate of the LO phonon at $10 \mathrm{~K}$, which will be discussed in detail in section 4.2. It is also clearly shown in the Figs. 3.9 (a) and (b), $E_{T H z}$ increases more abruptly with increasing electric field and its bipolar feature becomes pronounced.

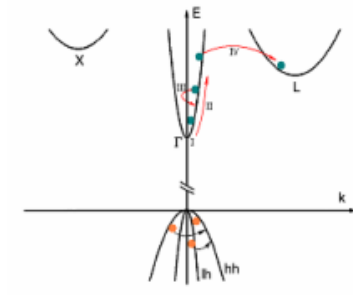

(a)
Ideal case: without scattering

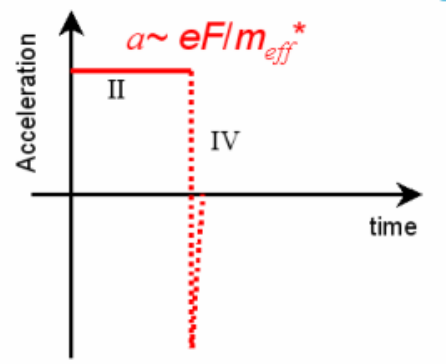

(b)

Fig. 3.10. (a) The band diagram of the GaAs; I: Electrons generated at the bottom of the $\Gamma$ valey; II: Ballistic acceleration; III: Intravalley scattering; IV: Intervalley scattering; (b) ideal acceleration of electrons in bulk GaAs

Taking advantage of the novel experimental method, invaluable information on nonequilibrium carrier transport in the femtosecond time range, which has previously been discussed only by numerical simulations, has been obtained. The present insights on the nonstationary carrier transport contribute to better understanding of device physics in existing high speed electron devices and, furthermore, to new design of novel ultrafast electromagnetic wave oscillators.

\section{Power dissipation spectrum under step electric field in Bulk GaAs in terahertz region}

It is well known that negative differential conductivities (NDCs) due to intervalley transfer appear in many of the compound semiconductors, such as GaAs, under high electric fields. NDCs are of practical importance, notably for its exploitation in microwave and ultrafast electromagnetic wave / THz wave oscillators [47]. However, since such NDC gain has a finite bandwidth, it gives intrinsic upper frequency limit to ultrafast electromagnetic wave oscillators [48]. For this reason, a number of works have been done to investigate the mechanism, which limits the bandwidth of the gain, and found that it is mainly controlled by the energy relaxation time [49]-[51]. Figure 3.11 shows the real and imaginary parts of the 
differential mobility spectra, $\operatorname{Re}[\mu(\omega)]$ and $\operatorname{Im}[\mu(\omega)]$, in bulk GaAs for various electric fields at $300 \mathrm{~K}$ calculated by using Monte Carlo method [52]. Monte Carlo results predicted that the real part of the negative differential mobility (i.e., gain) can persist up to a few hundred $\mathrm{GHz}$, as seen in Fig. 3.11. Together with theoretical works, efforts have been devoted to push the high frequency limit into the $\mathrm{THz}$ frequency range. Gunn and IMPATT diodes have already been operated at frequencies of $100-200 \mathrm{GHz}$ [53]-[55]. However, in those experiments, high frequency operation was not possible due to electronic circuits; i.e., the cutoff frequency, $v_{c}$, is mainly governed by stray capacitance, inductance, and more fundamentally by the length of the samples, not by the material properties themselves. Therefore, it is extremely important to characterize the gain bandwidth of materials in the $\mathrm{THz}$ range.
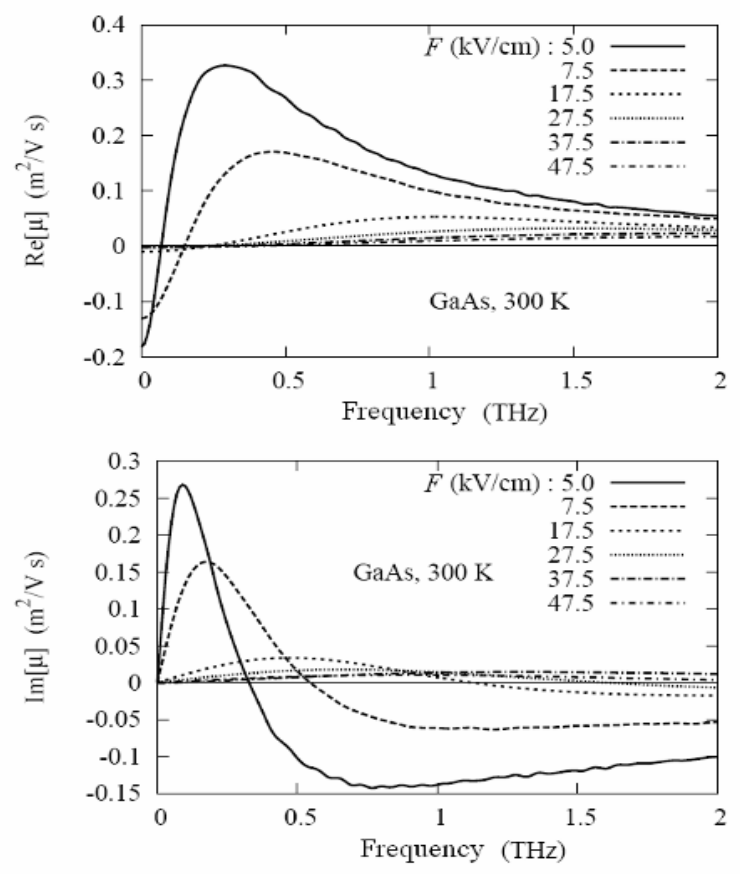

Fig. 3.11. The real and imaginary parts of the differential mobility spectra, $\operatorname{Re}[\mu(\omega)]$ and $\operatorname{Im}[\mu(\omega)]$, in bulk GaAs in THz range for various electric field at $300 \mathrm{~K}$ calculated by using Monte Carlo method [52]

The time domain $\mathrm{THz}$ spectroscopy has provided us with a unique opportunity to observe the motion of electron wave packet in the sub-picosecond time range and inherently measuring the response of the electron system to the applied bias electric field [11][12][15]. The Fourier spectra of the $\mathrm{THz}$ emission give us the power dissipation spectra under step electric field in $\mathrm{THz}$ range, from which we can find the gain region of material in $\mathrm{THz}$ range. In this section, we present the measured $\mathrm{THz}$ radiation from the intrinsic bulk GaAs under strong biased electric field. The power dissipation spectra under step electric field in $\mathrm{THz}$ range have been obtained by using the Fourier transformation of the time domain $\mathrm{THz}$ 
traces [17] [18]. From the power dissipation spectra, the cutoff frequency for negative power dissipation of the bulk GaAs has also been found. It is found that the cutoff frequency for the gain gradually increases with increasing electric fields up to $50 \mathrm{kV} / \mathrm{cm}$ and saturates at around $1 \mathrm{THz}$ at $300 \mathrm{~K} / 750 \mathrm{GHz}$ at $10 \mathrm{~K}$. We also investigated the temperature dependence of cutoff frequency for negative power dissipation, from which we find that this cutoff frequency is governed by the energy relaxation process of electrons from the $\mathrm{L}$ valley to the $\Gamma$ valley via successive optical phonon emission.

\subsection{Power dissipation spectrum under step electric field in GaAs}

Firstly, we recall the fact that the time domain $\mathrm{THz}$ emission experiments inherently measure the step response of the electron system to the applied electric field, as described in more detail in (Shimad et al., 2003). In the $\mathrm{THz}$ emission experiment, we first set a DC electric field, $F$, in the samples and, then, shoot a femtosecond laser pulse to the sample at $t$ $=0$ to create a step-function-like carrier density, $n \Theta(t)$, as shown in Fig. 3.12 (a)-(c). Subsequently, $\mathrm{THz}$ radiation is emitted from the accelerated photoexcited electrons [Fig. 3.12 (d)]. Now, by using our imagination, we view the experiment in a different way. Let's perform the following thought experiment, as shown in Figs. 3.12 (e)-(h); we first set electrons in the conduction band under a flat band condition [Fig. 3.12 (f)] and, at $t=0$, suddenly apply a step-function-like bias electric field, $\mathrm{F \Theta}(\mathrm{t})$ [Fig. $3.12(\mathrm{~g})$ ]. We notice that electrons in the thought experiment emit the same $\mathrm{THz}$ radiation as in the actual experiment [Fig. $3.12(\mathrm{~h})$ ]. This fact means that what we do in our femtosecond laser pulse measurement is equivalent to application of a step-function-like electric field to electrons. The step like electric field can be described as,

$$
F(t)=F_{0} \Theta(t)= \begin{cases}0 & (t<0) \\ F_{0} & (t>0)\end{cases}
$$

By noting this important implication, the power dissipation spectra under step-function-like electric fields in $\mathrm{THz}$ range can be obtained from the thought experimental scheme, as shown in the following;

In time domain, the power density is defined as $P(t)=\frac{\partial \varepsilon}{\partial t}=J(t) F(t)$, i.e., power density equals the product of the current density, $J(t)$, and electric field, $F(t)$. If the direction of $J(t)$ is the same as the direction of $F(t)$, this gives a Joule heating. However, if $\mathrm{P}(\mathrm{t})$ is negative, this is a gain. Similarly, if the power dissipation spectrum, $S(\omega)=\frac{\partial \varepsilon}{\partial \omega}$, is negative at frequency $\omega$, it means the system gains the energy at this frequency; i.e., gain in the frequency domain. The power dissipation spectrum, $S(\omega)$, is closely related to the differential conductivity spectrum, as

$$
S(\omega)=\sigma(\omega)|F(\omega)|^{2}
$$

in the linear response regime. However, this formulation which use small signal conductivity, $\sigma(\omega)$, is not strictly correct in nonlinear response situation. Therefore, we will avoid the formulation using $\sigma(\omega)$ and derive the power dissipation spectrum from the time domain $\mathrm{THz}$ data. 


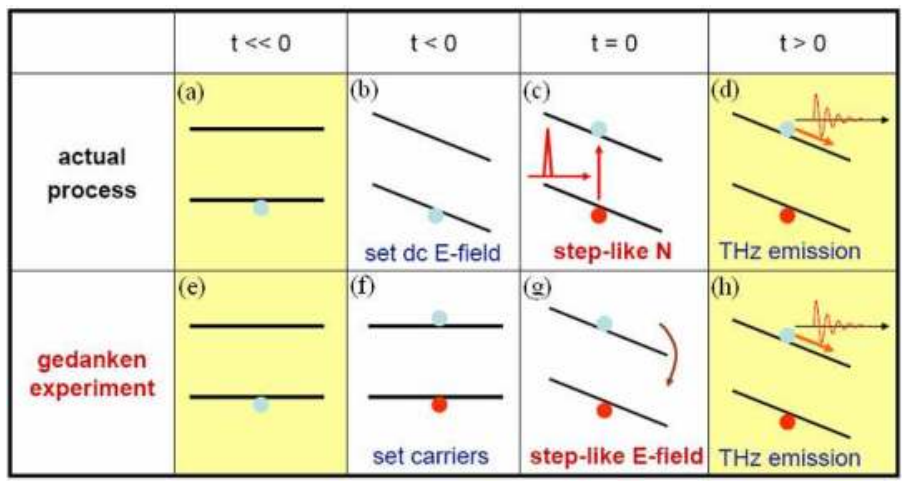

Fig. 3.12. The comparison between the real experiment [(a)-(d)] and thought experiment [(e)(h)]. Electrons in the thought experiment emit the same $\mathrm{THz}$ radiation as in the actual experiment, which means the time domain $\mathrm{THz}$ emission experiments inherently measure the step response of the electron system to the applied electric field

Mathematically, the power dissipation, $\varepsilon$, can be expressed as,

$$
\varepsilon=\int_{-\infty}^{+\infty} P(t) d t=V \int_{-\infty}^{+\infty} J(t) F(t) d t
$$

where $V$ is the volume of the sample, $J(t)$ and $F(t)$ are the time dependent current density and applied electric field, respectively. On the other hand, the energy can also be expressed in the frequency domain as,

$$
\varepsilon=V \int_{-\infty}^{+\infty} J(t) F(t) d t=\frac{V}{2 \pi} \int_{-\infty}^{+\infty} J(\omega) \bar{F}(\omega) d \omega,
$$

where $J(\omega)$ and $F(\omega)$ are the Fourier spectra of $J(t)$ and $F(t)$, respectively. Then, the power dissipation spectrum is obtained as,

$$
S(\omega)=\frac{\partial \varepsilon}{\partial \omega}=\frac{V}{2 \pi} J(\omega) \bar{F}(\omega)
$$

From simple mathematics, $J(\omega)$ can be obtained from the Fourier transformation of the time domain $\mathrm{THz}$ traces as,

$$
\begin{aligned}
J(\omega) & =\int_{-\infty}^{+\infty} J(t) \exp (-i \omega t) d t \propto \int_{-\infty}^{+\infty}\left[\int_{-\infty}^{t} E_{\mathrm{THz}}(t) d t\right] \exp (-i \omega t) d t \\
& =\frac{1}{i \omega} \int_{-\infty}^{+\infty} E_{\mathrm{THz}}(t) \exp (-i \omega t) d t+\pi E_{\mathrm{THz}}(0) \delta(\omega)=\frac{1}{i \omega} E_{\mathrm{THz}}(\omega)+\pi E_{\mathrm{THz}}(0) \delta(\omega)
\end{aligned}
$$

As mentioned in section 4.3, the creation of step-function-like carrier density by femtosecond laser pulses in the actual experiment can be replaced with the application of step-function-like electric field in the thought experiment scheme, then, the $F(\omega)$ can be expressed as,

$$
F(\omega)=\int_{-\infty}^{+\infty} F(t) \exp (-i \omega t) d t=F_{0}\left[\frac{1}{i \omega}+\pi \delta(\omega)\right]
$$


where $F_{0}$ is the magnitude of the internal electric field applied on the sample.

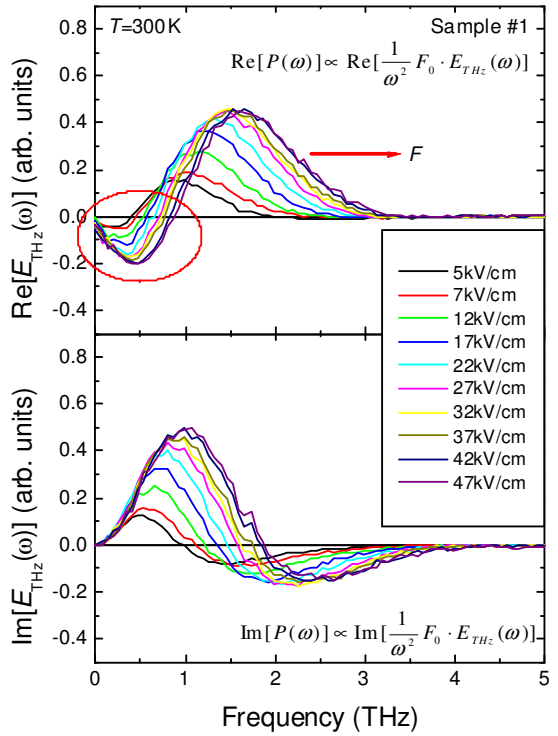

(a)

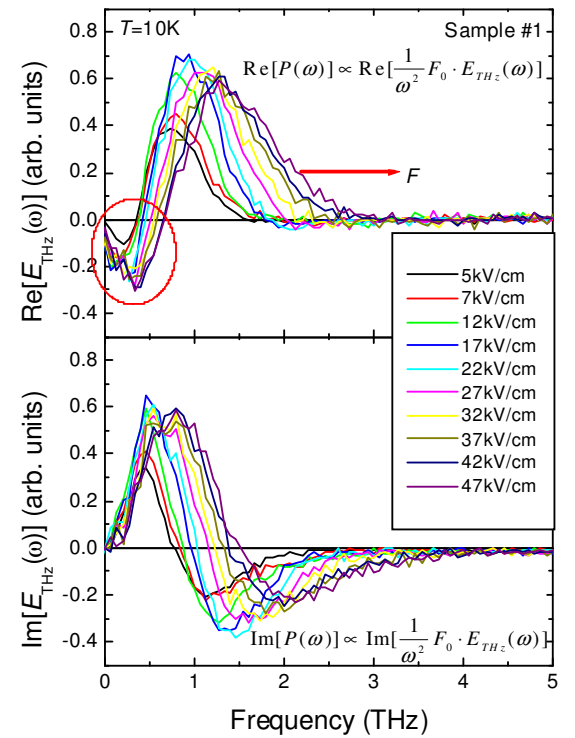

(b)

Fig. 3.13. The $\operatorname{Re}\left[E_{T H z}(\omega)\right]$ and $\operatorname{Im}\left[E_{T H z}(\omega)\right]$, which are proportional to the real and the imaginary parts of the power dissipation spectra in bulk GaAs for various $\mathrm{F}$ at $300 \mathrm{~K}$ and $10 \mathrm{~K}$ come from the Fourier transformation of THz traces emitted from sample \#1, an $m-i-n$ diode sample with a $1 \mu$ m-thick intrinsic GaAs layer

Simply substituting Eq. (3.19) and (3.20) into Eq. (3.18), the power dissipation spectrum under step-function-like electric field can be written as,

$$
S(\omega)=\frac{F_{0}}{\omega^{2}} E_{\mathrm{THz}}(\omega) \quad \text { for } \omega \neq 0
$$

Then, we can obtain an important message from Eq. (3.21) that the real and imaginary part of the Fourier spectra of $E_{T H z}(t)$ (i.e., $\operatorname{Re}\left[E_{T H z}(\omega)\right]$ and $\operatorname{Im}\left[E_{T H z}(\omega)\right]$ ) are proportional to $\operatorname{Re}[S(\omega)]$ and $\operatorname{Im}[S(\omega)]$, respectively.

From the discussion above, we know from the measured temporal waveforms of $\mathrm{THz}$ electric fields emitted from GaAs samples shown in Figs. 3.9, the power dissipation spectra in intrinsic bulk GaAs under step-function-like electric fields can be determined by using Fourier transformation of $E_{T H z}(t)$. Figures $3.13(\mathrm{a})$ and $(\mathrm{b})$ show $\operatorname{Re}\left[E_{T H z}(\omega)\right]$ and $\operatorname{Im}\left[E_{T H z}(\omega)\right]$ for sample \#1, a metal-intrinsic-n type semiconductor $(m-i-n)$ diode sample with a $1 \mu \mathrm{m}$ thick intrinsic GaAs layer under various electric fields at $300 \mathrm{~K}$ and $10 \mathrm{~K}$, respectively. In the Fourier transformation process, the position of $t=0$ is very crucial. Here, we determined $t=$ 0 by using the maximum entropy method [44]-[46]. $t=0$ can be determined within an error of $\pm 30 \mathrm{fs}$, which is limited by the time interval of the recorded points. The phase misplacement can be written as $\Delta \theta=\omega \Delta t$. Therefore, the phase error is estimated within an error of $< \pm \pi / 7$, assuming that the frequency of the signal, $\omega$, is mainly around $\sim 2.5 \mathrm{THz}$. 


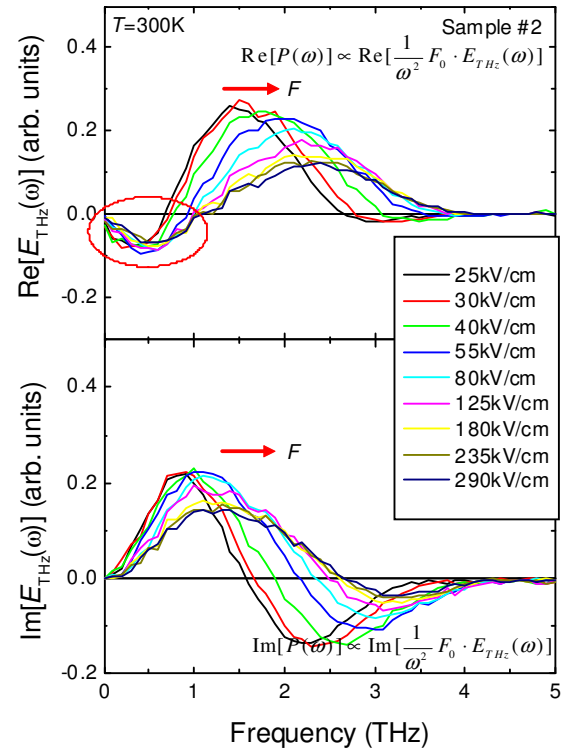

(a)

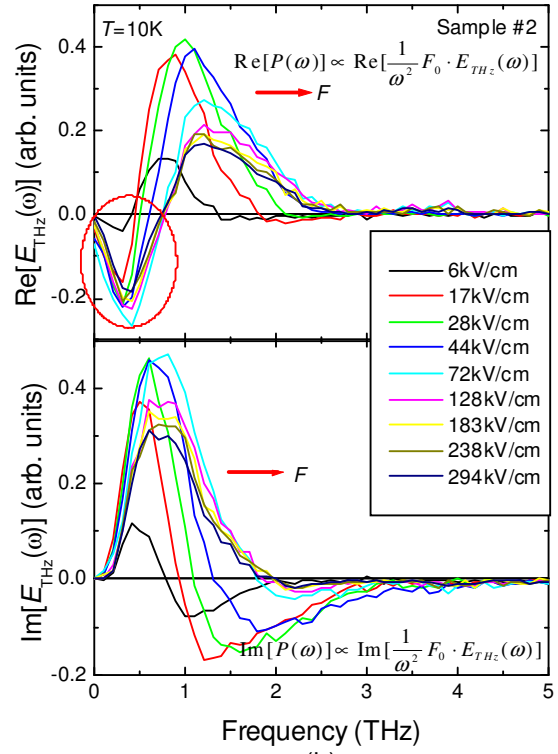

(b)

Fig. 3.14. The $\operatorname{Re}\left[E_{T H z}(\omega)\right]$ and $\operatorname{Im}\left[E_{T H z}(\omega)\right]$, which are proportional to the real and the imaginary parts of the power dissipation spectra in bulk GaAs for various $\mathrm{F}$ at $300 \mathrm{~K}$ and $10 \mathrm{~K}$ come from the Fourier transformation of THz traces emitted from sample \#2, an $m-i-n$ diode sample with a $180 \mathrm{~nm}$-thick intrinsic GaAs layer

From $\operatorname{Re}\left[E_{T H z}(\omega)\right]$ plotted in Fig. 3.13, we can see the negative region of $\operatorname{Re}[S(\omega)]$ persists up to several hundred $\mathrm{GHz}(\sim 800 \mathrm{GHz}$ at $47 \mathrm{kV} / \mathrm{cm}$ at $300 \mathrm{~K})$, indicating that GaAs has an intrinsic gain region up to the $\mathrm{THz}$ range. This frequency is much higher than the gain region predicted by Monte Carlo simulation shown in Fig. 3.11. The negative power dissipation turns to positive as the frequency increases. Comparing with $\operatorname{Re}\left[E_{T H z}(\omega)\right]$ at $10 \mathrm{~K}$ and $300 \mathrm{~K}$ shown in Figs. 3.13 (a) and (b), we find magnitude of $S(\omega)$ at $10 \mathrm{~K}$ is larger and the width is narrower than those at $300 \mathrm{~K}$ at same applied electric fields.

In the case of collisions with phonons in the $\Gamma$ valley, each collision redirects the momentum and restrains the drift velocity. Furthermore, intervalley scattering from the $\Gamma$ valley to the $L$ valley requires phonon with a large wave vector, and group theoretical selection rules show that the longitudinal optical (LO) phonon is the only one allowed because the $\mathrm{L}$ valley minimum lies on the edge of the Brillioun zone. Because the probability of LO phonon emission is proportional to $<\mathrm{N}_{\mathrm{LO}}+1>\left(\mathrm{N}_{\mathrm{LO}}\right.$ is the thermal equilibrium phonon population per unit volume; $\left.N_{\mathrm{LO}}=\frac{1}{\exp \left(\hbar \omega_{\mathrm{LO}} / k_{\mathrm{B}} T\right)-1}\right)$, which is temperature dependent, we expect the scattering time is shorter at higher temperatures. The calculation result by Ruch and Fawcett's also showed different magnitude of drift velocity at low and high temperatures [56].

Furthermore, to investigate the power dissipation spectra under extremely large electric fields, we measured the time domain $\mathrm{THz}$ traces emitted from sample \#2, an $m-i-n$ diode with a $180 \mathrm{~nm}$-thick intrinsic GaAs layer, at extremely high electric fields at $300 \mathrm{~K}$ and $10 \mathrm{~K}$. Figures 3.14 (a) and (b) shows the real and imaginary parts of the Fourier spectra of the time 
domain $\mathrm{THz}$ traces emitted from samples \#2. We can see even at very high electric fields, for example, $F=300 \mathrm{kV} / \mathrm{cm}$, the negative $\operatorname{Re}\left[E_{T H z}(\omega)\right]$ still exists at low frequency region. At high frequency, the $\operatorname{Re}\left[E_{\mathrm{THz}}(\omega)\right]$ changes its sign from negative to positive.

\subsection{The cutoff frequency for negative power dissipation in GaAs}

\subsubsection{Electric field dependence of the cutoff frequency}

From the real part of the power dissipation spectra, we can find the cutoff frequencies for the gain region, $v_{c}$, at various $F$. The solid circles and the solid triangles in Fig. 3.15 show $v_{c}$ obtained from sample \#1 as a function of $\mathrm{F}$ at $300 \mathrm{~K}$ and $10 \mathrm{~K}$. The open circles and the open triangles correspond to $v_{c}$ obtained from sample \#2 at $300 \mathrm{~K}$ and $10 \mathrm{~K}$, respectively. The cutoff frequency increases with increasing $F$ for $F<50 \mathrm{kV} / \mathrm{cm}$. However, above $50 \mathrm{kV} / \mathrm{cm}$, $v_{c}$ starts to saturate. The sharp kink of $v_{c}$ at $50 \mathrm{kV} / \mathrm{cm}$ shown in Fig. 3.15 strongly suggests that this is a very critical electric field. This point will be discussed in section 4.3.

\subsubsection{Temperature dependence of the cutoff frequency}

To clarify the mechanism of the cutoff frequency, $v_{c}$, we also investigated the temperature dependence of $v_{c}$. Figure 3.16 shows the temperature dependence of $v_{c}$ obtained for sample $\# 1$ at $7 \mathrm{kV} / \mathrm{cm}$ and $27 \mathrm{kV} / \mathrm{cm}$ by solid triangles and circles, respectively. We can clearly see that the temperature dependence of $v_{c}$ agrees with that of the emission rate of LO phonons, $<\mathrm{N}_{\mathrm{LO}}+1>$ (plotted by a dashed line in Fig. 3.16). The agreement between the experimental results and the LO phonon emission rate strongly suggests that $v_{c}$ is governed by the energy relaxation process of electrons from the $L$ valley to the $\Gamma$ valley via optical phonon emission. At lower temperature, $\mathrm{LO}$ phonon emission rate decreases and a longer energy relaxation time is expected, which results in a lower $v_{c}$ at $10 \mathrm{~K}$ than at $300 \mathrm{~K}$, as shown in Fig. 3.15.

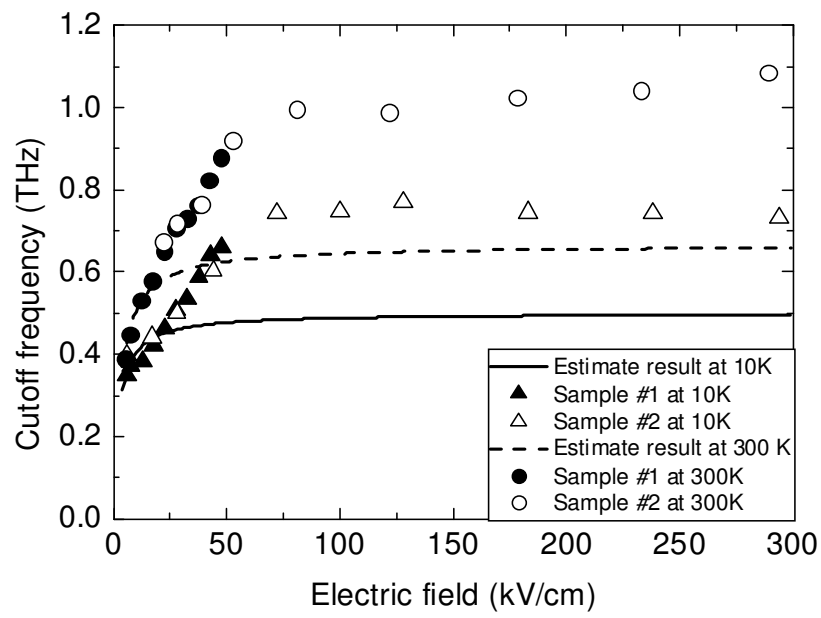

Fig. 3.15. The cutoff frequencies of the negative region of $\operatorname{Re}\left[E_{T H z}(\omega)\right]$ obtained for sample \#1, as a function of the electric field $F$ at $300 \mathrm{~K}$ and $10 \mathrm{~K}$ are shown by solid circles and triangles, respectively. The open circles and triangles correspond to the cutoff frequencies obtained from sample \#2, at $300 \mathrm{~K}$ and $10 \mathrm{~K}$, respectively. The estimated cutoff frequencies are also plotted by a dashed line and a solid line at $300 \mathrm{~K}$ and $10 \mathrm{~K}$, respectively 


\subsection{Mechanism for the cutoff frequency}

When a small step-function electric field is added on a large DC electric field, the electron velocity is predominantly redirected, which causes a general increase of the kinetic energy, or a heating, of the electron system until the mean collision rate with the lattice has risen sufficiently to balance the increasing supply of energy to the electrons from the electric field. The characteristic time for this thermal readjustment is known as the energy relaxation time, which is the limiting process for the upper frequency limit of transferred electron devices [49]. However, the mechanism for the cutoff frequency for gain, $v_{c}$, under extremely nonequilibrium condition has been still argued for more than 30 years.

\subsubsection{Energy relaxation and momentum relaxation times govern $v_{c}$.}

Monte Carlo simulation has been used as a tool to estimate $v_{c}$ [52] [57], as shown in Fig. 3.11. Gruzinskis [58] and Reggiani, et al. [52] calculated the energy relaxation time $\tau_{\varepsilon}$ by Monte Carlo simulation and found that $\tau_{\varepsilon}$ is $\sim 1.5$ ps, as shown in Fig. 3.17. Also, they discussed that $v_{c}$ is determined by both energy relaxation time $\tau_{\varepsilon}$ and momentum relaxation time $\tau_{v}\left(\tau_{v}\right.$ is field dependent and $\tau_{v}<<\tau_{\varepsilon}$ as shown in Fig. 3.17) [58]. The calculated $v_{c}$ is below 500 $\mathrm{GHz}$, as shown in Fig. 3.11, which is much lower than $v_{c}$ obtained by our experiment.

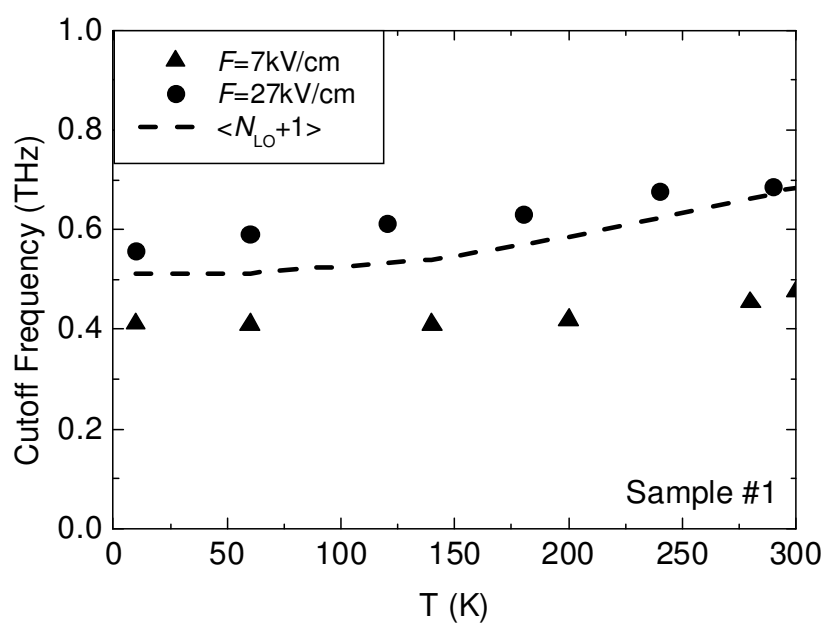

Fig. 3.16. The cutoff frequencies of the negative region of $\operatorname{Re}\left[E_{T H z}(\omega)\right]$ obtained for sample \#1, as a function of the temperature, at $7 \mathrm{kV} / \mathrm{cm}$ and $27 \mathrm{kV} / \mathrm{cm}$ are shown by solid triangles and circles, respectively. The reciprocal of the energy relaxation time due to the LO scattering in $\Gamma$ valley as a function of temperature $T$ is also plotted by a dashed line

\subsubsection{Relaxation process of electrons in the $L$ valley governs $v_{c}$.}

Another model is that the energy relaxation time is governed by relaxation process of electrons in the L valley [50]. The electrons start to be accelerated at the bottom of the $\Gamma$ valley, scattered into the $L$ valley, relax and dwell there, and, then, scattered back to the $\Gamma$ valley. In this model, $v_{c}$ is mainly governed by relaxation time of electrons in the $\mathrm{L}$ valley and estimated to be $\sim 200 \mathrm{GHz}$, which is also much lower than our experimental result. 


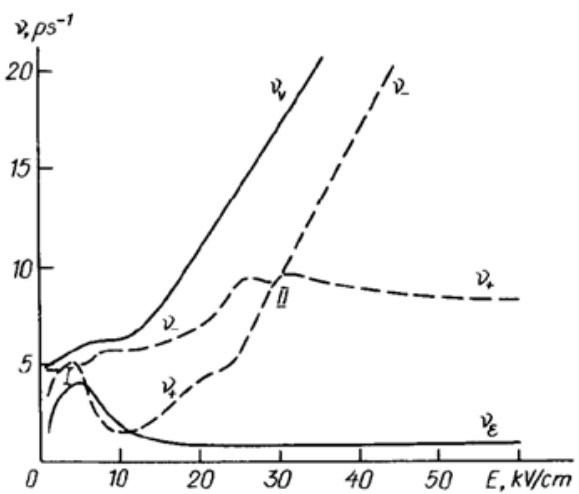

Fig. 3.17. The calculated energy and velocity relaxation rates (solid line) in $n$-InP [58]

\subsubsection{Relaxation process of electrons in the $\Gamma$ valley governs $v_{c}$.}

The temperature dependence of $v_{c}$, strongly suggests that $v_{c}$ is mainly governed by the energy relaxation process of electrons from the $L$ valley to the $\Gamma$ valley via successive optical phonon emission. Here, we roughly estimate $v_{c}$.

In the $\varepsilon-k$ diagram, the electrons experience a cyclic motion in the $k$ space; i.e., be accelerated from the bottom of the $\Gamma$ valley, go to the $L$ valley by intervalley scattering, be scattered back, and then relax to the bottom of the $\Gamma$ valley again, as shown in Fig. 3.18 (a).

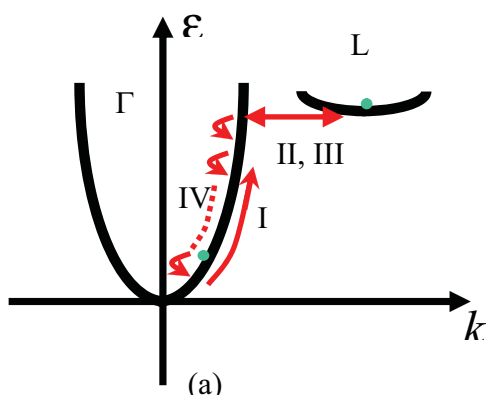

(a)

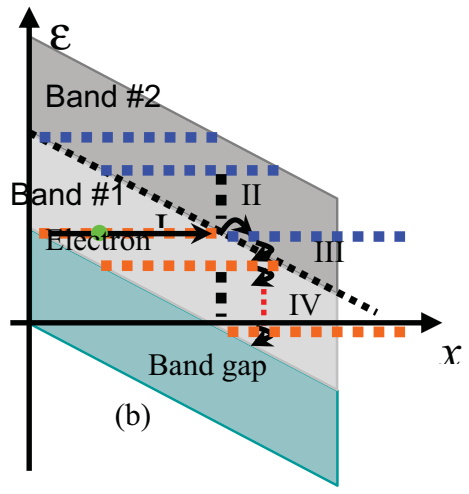

Fig. 3.18. (a) Electrons experience a cyclic motion in the $k$ space; I: acceleration from the bottom of $\Gamma$ valley; II: intervalley scattering to the L valley; III: intervalley scattering back to the $\Gamma$ valley; IV: relaxation to the bottom of the $\Gamma$ valley; (b) the real space picture; I: acceleration in the band \#1 (associated with the $\Gamma$ valley); II: reach the edge of the band \#2 (the L valley related states) and experience intervalley scattering; III: scattering back; IV: relaxation to the bottom of the conduction band

For this order estimation, we assume electrons are ballistically accelerated by the electric field $F$ in the $\Gamma$ valley. In addition, we also neglect the acoustic scattering, $e$-e scattering. Assuming the simple Newton's law 


$$
\frac{\hbar d k}{d t}=-e F
$$

where $e$ is the elementary charge, $\hbar=h / 2 \pi$ ( $h$ is the Planck constant), $k$ is the wave vector of electrons, the time for the acceleration process can be estimated by assuming the energy separation between the $\Gamma$ minimum and the bottom of $\mathrm{L}$ valley $\varepsilon_{\Gamma-\mathrm{L}}=0.29 \mathrm{eV}$. The subsequent intervalley scattering time is about $50 \mathrm{fs}$ [59].

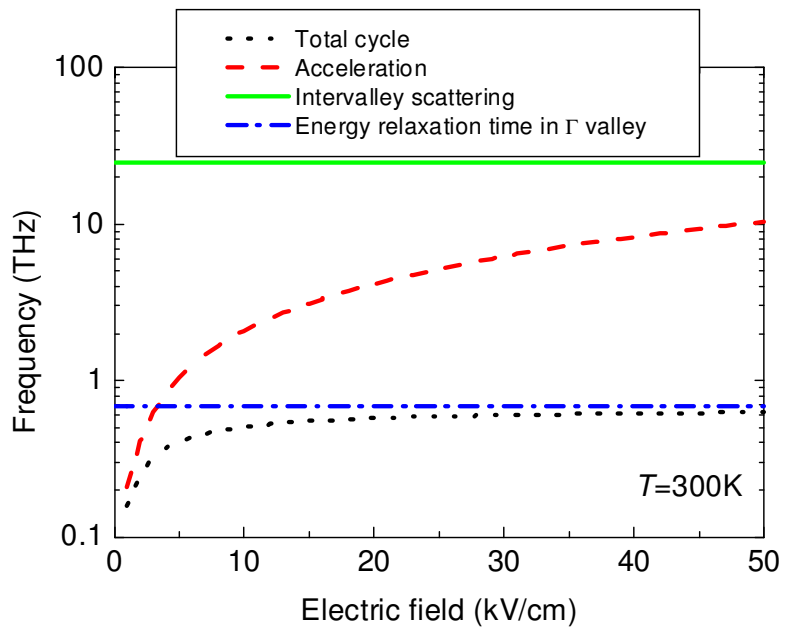

Fig. 3.19. The estimated cutoff frequencies of the negative region of $\operatorname{Re}\left[E_{T H z}(\omega)\right]$ as a function of the electric field $F$ at $300 \mathrm{~K}$ are shown by black dashed line. The red dashed line, green solid line and blue dash-dotted line correspond to ballistic acceleration process, the intervalley transfer process and the energy relaxation process in $\Gamma$ valley, at $300 \mathrm{~K}$, respectively

The energy relaxation process in the $\Gamma$ valley is dominated by the $\mathrm{LO}$ phonon scattering. Since the LO phonon energy is $36.5 \mathrm{meV}, 8 \mathrm{LO}$ phonons must be successively emitted in the process for electrons to relax from the bottom of the $\mathrm{L}$ valley to the bottom of $\Gamma$ valley $\left(\varepsilon_{\Gamma-\mathrm{L}}=\right.$ $0.29 \mathrm{eV})$. The relaxation time for one polar scattering process by LO phonon emission in bulk is expressed [49],

$$
\begin{aligned}
& \frac{1}{\tau_{(e)}(\hat{k})}=\frac{2 \pi}{\hbar} \frac{1}{(2 \pi)^{3}} \frac{e^{2} \hbar \omega_{\mathrm{LO}}}{2 \varepsilon_{0}}\left\langle N_{\mathrm{LO}}+1\right\rangle\left(\frac{1}{\varepsilon_{\infty}}-\frac{1}{\varepsilon_{r}}\right) \frac{m_{e f f}{ }^{*}}{\hbar^{2} \sqrt{|\hat{k}|^{2}-\frac{2 m_{e f f}{ }^{*} \hbar \omega_{\mathrm{LO}}}{\hbar^{2}}}} \times \\
& \int_{0}^{+\infty} \int_{0}^{\pi} \int_{0}^{2 \pi}\left|k^{\prime}\right|^{2} \sin \theta \frac{1}{\left|\hat{k}^{\prime}-\hat{k}\right|^{2}} \delta\left(|\hat{k}|^{\prime}-\sqrt{|\hat{k}|^{2}-\frac{2 m_{e f f}{ }^{*} \hbar \omega_{\mathrm{LO}}}{\hbar^{2}}}\right) d|\hat{k}|^{\prime} d \theta d \phi
\end{aligned}
$$

where $\varepsilon_{0}$ is the dielectric permittivity of the vacuum, $\varepsilon_{\mathrm{s}}$ is the static dielectric constant, $\varepsilon_{\infty}$ is the high frequency dielectric constant, $\mathrm{N}_{\mathrm{LO}}$ is the thermal equilibrium phonon population in per unit volume, $\hbar=h / 2 \pi$ ( $h$ is the Planck constant), $\omega_{\text {LO }}$ is the frequency of LO phonon, $m_{\text {eff }}^{*}$ is the effective mass of electron, and $k, k^{\prime}$ are the wave vector of electrons before and after scattering, respectively. 
By using Eq. (3.23), the energy relaxation time in the $\Gamma$ valley is estimated to be 1.46 ps at 300 $\mathrm{K}$ and 1.95 ps at $10 \mathrm{~K}$, by summing up emission times of $8 \mathrm{LO}$ phonons. The temperature dependence of the energy relaxation time is due to the temperature dependence of the phonon emission process, $<\mathrm{N}_{\mathrm{LO}}+1>$. The estimated cutoff frequency, $v_{c}$, at $300 \mathrm{~K}$, are plotted in Fig. 3.19 [ballistic acceleration (dashed line), intervalley transfer (solid line), energy relaxation (dash-dotted line), and the total (dotted line)]. From this estimation, it is concluded that the energy relaxation process in the $\Gamma$ valley takes the longest time and governs the upper frequency limitation for the gain. Furthermore, the estimated results are also plotted by a dashed line and a straight line for $300 \mathrm{~K}$ and $10 \mathrm{~K}$, respectively, in Fig. 3.15 for the comparison with the experimental results.

As shown in Fig. 3.15, the overall trend of this estimation of $v_{c}$ is the same as our experimental results. However, at very high electric fields, the magnitude is off by $40 \%$. The discrepancy between experiment and calculation may come from the field dependent energy relaxation time of electrons in the $\Gamma$ valley as calculated by Fischetti [59]. Fischetti's calculation results show that at very high electric fields, the energy relaxation process is two times faster than that at low electric fields [59]. Then, the dash-dotted line of Fig. 3.19 shifts to higher frequency range, which results in higher $v_{\mathrm{c}}$.

Under very high electric fields, Wannier Stark (WS) ladder may be formed, although it has never been observed experimentally. The cyclic motion of electrons in the $\mathrm{k}$ space can be expressed in the real space as Fig. 3.18 (b). Electrons are created in the WS ladder states immediately after short pulsed photoexcitation under DC electric field $F$, and accelerated in the conduction band (band \#1 in Fig. 3.18 (b)), which corresponds to the ballistic acceleration in the $\Gamma$ valley. When they reach the edge of the band $\# 1$, they are scattered into the states in the band \#2 (L valley related states, as shown in Fig. 3.18 (b)). After electrons dwell in the upper states, they are scattered downward (intervalley transfer process), and then relax to the bottom of the conduction band (energy relaxation in the $\Gamma$ valley process).

Based on our experimental data, we conclude that the cutoff frequency is governed by the energy relaxation process of electrons in the $\Gamma$ valley via successive optical phonon emission. In our estimation, we neglect scattering while electrons are accelerated by the electric field in the band \#1. This scattering in the acceleration process makes the sharp kink in the cutoff frequency, $v_{\mathrm{c}}$, around $50 \mathrm{kV} / \mathrm{cm}$, as shown in Fig. 3.15. In the low electric field range $(F<50 \mathrm{kV} / \mathrm{cm})$, the number of events of LO phonon scattering decreases with increasing electric field $F$. Each scattering takes $\sim 0.13$ ps. However, in the high electric field range $(F>50 \mathrm{kV} / \mathrm{cm})$, electrons at the bottom of the conduction band can go into the band \#2 without experiencing any scattering. The mean free path of electrons can be estimated by,

$$
\lambda=\frac{1}{2} \frac{e F}{m_{e f f} *} \tau_{v}^{2},
$$

where the momentum relaxation time of electrons in band $\# 1$ ( $\Gamma$ valley) is about $\tau_{v} \sim 0.13$ ps, mainly limited by LO phonon scattering. The mean free path is calculated to be $\sim 60 \mathrm{~nm}$ at $F$ $=50 \mathrm{kV} / \mathrm{cm}$. On the other hand, the distance, $l$, for electrons starting from the bottom of conduction band to the bottom of band \#2 is estimated from,

$$
e F l=\varepsilon_{\Gamma-\mathrm{L}}=0.29 \mathrm{eV} .
$$


At $50 \mathrm{kV} / \mathrm{cm}, 1$ is calculated to be $\sim 60 \mathrm{~nm}$, almost the same as the mean free path of electrons. Therefore, we can conclude that when $F<50 \mathrm{kV} / \mathrm{cm}, v_{\mathrm{c}}$ increases with increasing $F$ because LO phonon scattering strongly affects the acceleration process, and that for $\mathrm{F}>50$ $\mathrm{kV} / \mathrm{cm}, v_{\mathrm{c}}$ is almost $F$ independent because no scattering takes place in the acceleration process. The illustration of this situation is shown in Figs. 3.20.

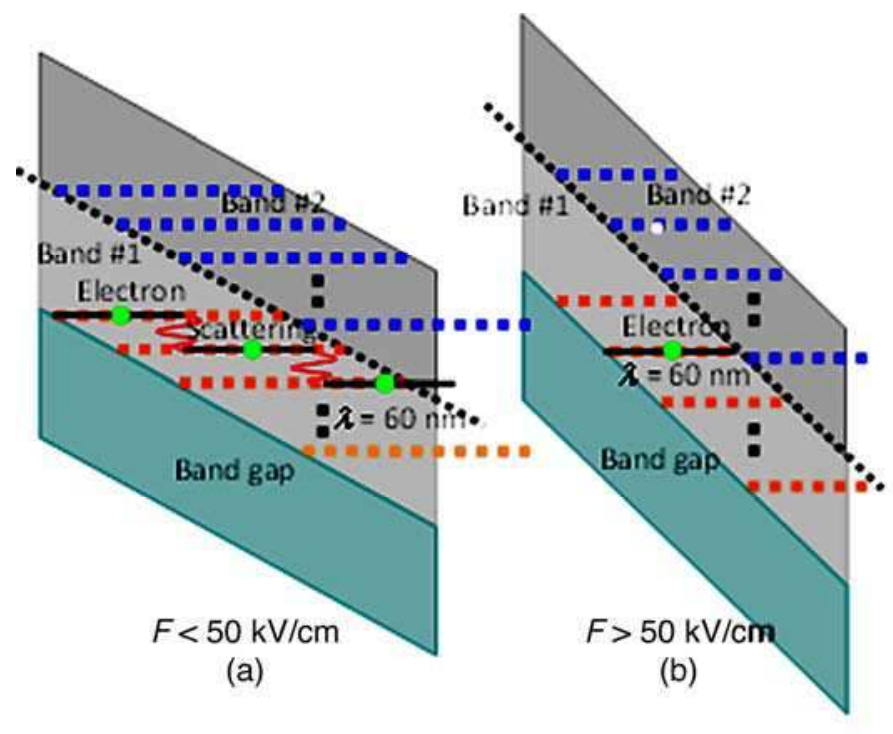

Fig. 3.20. (a) For $F<50 \mathrm{kV} / \mathrm{cm}$, LO phonon scattering strongly affects the acceleration process; (b) $F>50 \mathrm{kV} / \mathrm{cm}$, no scattering takes place in the acceleration process

The other possible explanation is that the strong band mixing gives the sharp kink in $v_{\mathrm{c}}$ around $50 \mathrm{kV} / \mathrm{cm}$, as shown in Fig. 3.15. For $F>50 \mathrm{kV} / \mathrm{cm}$, the tunneling probability for electrons in the band \#1 to the band \#2 is significantly enhanced (Zener tunneling). Such Zener tunneling process may limit the increase in $v_{\mathrm{c}}$.

In summary, the power dissipation spectra under step electric field in bulk GaAs in $\mathrm{THz}$ range have been investigated by Fourier transformation of the ultrafast electromagnetic waveforms/THz waveforms emitted from intrinsic bulk GaAs photoexcited by femtosecond laser pulses under strong bias electric fields. The cutoff frequency for the gain region, that is of practical importance, notably for its exploitation in ultrafast electromagnetic wave generators, has been also found. The cutoff frequency gradually increases with increasing electric field within $50 \mathrm{kV} / \mathrm{cm}$, saturates and reaches $1 \mathrm{THz}(300 \mathrm{~K})$ above $50 \mathrm{kV} / \mathrm{cm}$. Furthermore, illustrated by the electric and temperature dependence of the cutoff frequency, we also find out that this cutoff frequency is mainly dominated by the energy relaxation process of electrons from the $L$ to the $\Gamma$ valley via successive optical phonon emission.

\section{Conclusion}

Ultrafast electromagnetic waves emitted from semiconductors under high electric fields, which are closely related with ultrafast nonequilibrium transport of carriers in 
semiconductor, are of fundamental interest in semiconductor physics. Furthermore, clarifying carrier dynamics under extreme nonequilibrium conditions is also strongly motivated by the need to obtain information relevant for the design of ultrahigh speed devices and ultrafast electromagnetic wave emitters. It is, therefore, essential to understand nonequilibrium transport of carriers subjected to high electric fields in such devices. The time domain terahertz $(\mathrm{THz})$ spectroscopy gives us a unique opportunity of observing motions of electron wave packets in the sub-ps time range and measuring the response of electron systems to applied bias electric fields. By understanding the nonequilibrium transport of carriers in bulk GaAs, the intrinsic property of the negative differential conductivity (NDC) in the $\mathrm{THz}$ region, which means the gain, can be clarified.

In section 2, we describe the experimental technique of the time domain $\mathrm{THz}$ spectroscopy used in this work. Ultrafast carrier motion in the femtosecond time regime accompanies electromagnetic wave radiation that is proportional to $\mathrm{d} v / \mathrm{d} t$. Consequently, the investigation of such electromagnetic wave (or $\mathrm{THz}$ radiation) allows us a very unique opportunity of looking directly into the acceleration/deceleration dynamics of carriers in semiconductors.

In section 3, the femtosecond acceleration of carriers in bulk GaAs under very high electric fields, $F$, has been investigated by time domain $\mathrm{THz}$ spectroscopy. The observed ultrafast electromagnetic emission waveforms / THz emission waveforms have a bipolar feature; i.e., an initial positive peak and a subsequent negative dip. The initial positive peak has been interpreted as electron acceleration in the bottom of $\Gamma$ the valley in GaAs, where electrons have a light effective mass, while the subsequent negative dip has been attributed to intervalley transfer from the $\Gamma$ valley to the $X$ and $L$ valleys.

In section 4, we have investigated the gain due to intervalley transfer under high electric fields, which is of practical importance for its exploitation in ultrafast electromagnetic wave oscillators. The power dissipation spectra under step-function-like electric fields in $\mathrm{THz}$ range have been obtained by using the Fourier transformation of the time domain $\mathrm{THz}$ traces. From the power dissipation spectra, the cutoff frequencies for negative power dissipation (i.e., gain) in bulk GaAs have been determined. The cutoff frequency gradually increases with increasing electric field, $F$, for $F<50 \mathrm{kV} / \mathrm{cm}$, saturates and reaches $1 \mathrm{THz}$ $(300 \mathrm{~K})$ for $F>50 \mathrm{kV} / \mathrm{cm}$. Furthermore, from the temperature dependence of the cutoff frequency, we found that it is governed by the energy relaxation process of electrons from $\mathrm{L}$ to $\Gamma$ valley via successive optical phonon emission.

Finally, section 5 summarizes this chapter. As demonstrated in this chapter, taking advantage of the novel experimental method, invaluable information on nonequilibrium carrier transport in the femtosecond time range, which has previously been discussed only by numerical simulations, has been obtained. The present insights on the nonstationary carrier transport contribute to better understanding of device physics in existing high speed electron devices. Furthermore, the gain in GaAs due to electrons intervalley transfer under high electric fields obtained from the Fourier transformation of the time domain $\mathrm{THz}$ traces is also discussed, which is of practical importance for its exploitation in ultrafast electromagnetic wave oscillators.

\section{Acknowledgment}

The authors thank Mr. JunAn Zhu, a student from the University of Shanghai for Science and Technology, for editing the manuscript of this chapter. 


\section{References}

[1] M. van Exter, Ch. Fattinger, and D. Grischkowsky, Terahertz time-domain spectroscopy of water vapour, Opt. Lett. Vol. 14, No. 20, pp. 1128-1130 (1989).

[2] D. Grischkowsky, S. Keiding, M. van Exter, and Ch. Fattinger, Far-infrared time-domain spectroscopy with terahertz beams of dielectrics and semiconductors, J. Opt. Soc. Am. B, Vol. 7, No. 10, pp. 2006-2015 (1990).

[3] R. A. Cheville, and D. Grischkowsky, Far-infrared terahertz time-domain spectroscopy of flames, Opt. Lett., Vol. 20, No. 15, pp. 1646-1648 (1995).

[4] B. Ferguson, S. Wang, D. Gray, D. Abbott, and X.-C. Zhang, T-ray computed tomography, Opt. Lett., Vol. 27, No. 15, pp. 1312-1314 (2002).

[5] B. B. Hu, and M. C. Nuss, Imaging with terahertz waves, Opt. Lett. Vol. 20, No. 16, pp. 1716-1718 (1995).

[6] S.P. Mickan, A. Menikh, H. Liu, C.A. Mannella, R. MacColl, D. Abbott, J. Munch, and X.C. Zhang, Label-free bioaffinity detection using terahertz technology, Physics in Medicine and Biology (IOP), Vol. 47, No. 21, pp. 3789-3795 (2002).

[7] S.P. Mickan, D. Abbott, J. Munch, and X-C Zhang, Noise reduction in terahertz thin film measurements using a double modulated differential technique, Fluctuation and Noise Letters (Elsevier), Vol. 2, No. 1, pp. R13-R28 (2002).

[8] M. J. W. Rodwell, M. Urteaga, T. Mathew, D. Scott, D. Mensa, Q. Lee, J. Guthrie, Y. Betser, S. C. Martin, R. P. Smith, S. Jaganathan, S. Krishnan, S. I. Long, R. Pullela, B. Agarwal, U. Bhattacharya, L. Samoska, and M. Dahlstrom, Submicron scaling of HBTs, IEEE Trans. Electron Devices, Vol. 48, No. 11, pp. 2606-2624 (2001).

[9] M. Beck, D. Hofstetter, T. Aellen, J. Faist, U. Oesterle, M. Ilegems, E. Gini, and H. Melchior, Continuous Wave Operation of a Mid-Infrared Semiconductor Laser at Room Temperature, Science, Vol. 295, No. 5553, pp. 301-305 (2002).

[10] G. A. Mourou, C. V. Stancampiano, A. Antonetti, and A. Orszag, Picosecond microwave pulses generated with a subpicosecond laser-driven semiconductor switch, Appl. Phys. Lett. Vol. 39, No. 4, pp. 295-296 (1981).

[11] A. Leitenstorfer, S. Hunsche, J. Shah, M. C. Nuss, and W. H. Knox, Femtosecond Charge Transport in Polar Semiconductors, Phys. Rev. Lett., Vol. 82, No. 25, pp. 5140-5142 (1999).

[12] A. Leitenstorfer, S. Hunsche, J. Shah, M. C. Nuss, and W. H. Knox, Femtosecond highfield transport in compound semiconductors, Phys. Rev. B, Vol. 61, No. 24, pp. 16642-16652 (2000).

[13] G. Zhao, R. N. Schouten, N. van der Valk, W. T. Wenchebach, and P. C. M. Planken, Design and performance of a THz emission and detection setup based on a semiinsulating GaAs emitter, Rev. Sci. Instrum., Vol. 73, No. 4, pp. 1715-1719 (2002).

[14] N. Katzenellenbogen, and D. Grischkowsky, Efficient generation of $380 \mathrm{fs}$ pulses of THz radiation by ultrafast laser pulse excitation of a biased metal-semiconductor interface, Appl. Phys. Lett., Vol. 58, No. 3, pp. 222-224 (1991).

[15] M. Abe, S. Madhavi, Y. Shimada, Y. Otsuka, and K. Hirakawa, Transient carrier velocities in bulk GaAs: Quantitative comparison between terahertz data and ensemble Monte Carlo calculations, Appl. Phys. Lett., Vol. 81, No. 4, pp. 679-681 (2002). 
[16] Y. M. Zhu, T. Unuma, K. Shibata, and K. Hirakawa, Femtosecond acceleration of electrons under high electric fields in bulk GaAs investigated by time-domain terahertz spectroscopy, Appl. Phys. Lett., Vol. 93, No. 4, pp. 042116-042118 (2008).

[17] Y. M. Zhu, T. Unuma, K. Shibata, and K. Hirakawa, Power dissipation spectra and terahertz intervalley transfer gain in bulk GaAs under high electric fields, Appl. Phys. Lett., Vol. 93, No. 23, pp. $232102-232104$ (2008).

[18] Y. M. Zhu, X. X. Jia, L. Chen, D. W. Zhang, Y. S. Huang, B. Y. He, S. L. Zhuang, and K. Hirakawa, Terahertz power dissipation spectra of electrons in bulk GaAs under high electric fields at low temperature, Acta Phys. Sinica, Vol. 58, No. 4, pp. 26922695 (2009).

[19] D. H. Auston, K. P. Cheung, J. A. Valdmanis, and D. A. Kleinman, Cherenkov Radiation from Femtosecond Optical Pulses in Electro-Optic Media, Phys. Rev. Lett., Vol. 53, No. 16, pp. 1555-1558 (1984).

[20] Ch. Fattinger, and D. Grischkowsky, Point source terahertz optics, Appl. Phys. Lett., Vol. 53, No. 16, pp. 1480-1482 (1988).

[21] D. H. Auston, K. P. Cheung, and P. R. Smith, Picosecond photoconducting Hertzian dipoles, Appl. Phys. Lett., Vol. 45, No. 3, pp. $284-286$ (1984).

[22] A. P. DeFonzo, M. Jarwala, and C. R. Lutz, Transient response of planar integrated optoelectronic antennas, Appl. Phys. Lett., Vol. 50, No. 17, pp. 1155-1157 (1987).

[23] C. Johnson, F. J. Low, and A. W. Davidson, Germanium and germanium-diamond bolometers operated at 4.2 K, 2.0 K, 1.2 K, 0.3 K, and 0.1 K, Opt. Eng., Vol. 19, pp. 255-258 (1980).

[24] R. C. Jones, The ultimate sensitivity of radiation detectors, J. Opt. Soc. Am. Vol. 37, No. 11, pp. 879-890 (1947).

[25] D. R. Dykaar, T. Y. Hsiang, and G. A. Mourou, An application of picosecond electrooptic sampling to superconducting electronics, IEEE Trans. Magn., Vol. 21, No. 2, pp. 230-233 (1985).

[26] J. A. Valdmanis, and G. A. Mourou, Sub-Picosecond Electro-Optic Sampling: Principles and Applications, IEEE J. Quant. Electron., Vol. 22, No. 1, pp. 69-78 (1986).

[27] P. U. Jepsen, C. Winnewisser, M. Schall, V. Schya, S. R. Keiding, and H. Helm, Detection of THz pulses by phase retardation in lithium tantalite, Phys. Rev. E Vol. 53, No. 4, pp. 3052-3054 (1996).

[28] A. Nahata, D. H. Auston, T. F. Heinz, and C. Wu, Coherent detection of freely propagating terahertz radiation by electro-optic sampling, Appl. Phys. Lett., Vol. 68, No. 2, pp. 150-152 (1996).

[29] Q. Wu, and X.-C. Zhang, Free-space electro-optic sampling of terahertz beams, Appl. Phys. Lett. Vol. 67, No. 24, pp. 3523-3525 (1995).

[30] G. Gallot, and D. Grischkowsky, Electro-optic detection of terahertz radiation, J. Opt. Soc. Am. B, Vol. 16, No. 8, pp. 1204-1212 (1999).

[31] A. Leitenstrofer, S. Hunsche, J. Shah, M. C. Nuss, and W. H. Know, Detectors and sources for ultrabroadband electro-optic sampling: Experiment and theory, Appl. Phys. Lett., Vol. 74, No. 11, pp. 1516-1518 (1999).

[32] Z. G. Lu, P. Campbell, and X.-C. Zhang, Free-space electro-optic sampling with a highrepetition-rate regenerative amplified laser, Appl. Phys. Lett., Vol. 71, No. 5, pp. 593595 (1997). 
[33] C. Winnewisser, P. U. Jepsen, M. Schall, V. Schya, and H. Heim, Electro-optic detection of $\mathrm{THz}$ radiation in $\mathrm{LiTaO}_{3}, \mathrm{LiNbO}_{3}$ and ZnTe, Appl. Phys. Lett., Vol. 70, No. 23, pp. 3069-3071 (1997).

[34] Q. Wu, and X.-C. Zhang, Electrooptic sampling of freely propagating terahertz fields, Opt. Quant. Electron., Vol. 28, No. 7, pp. 945-951 (1996).

[35] Q. Wu, M. Litz, and X.-C. Zhang, Broadband detection capability of ZnTe electro-optic field detectors, Appl. Phys. Lett., Vol. 68, No. 21, pp. 2924-2926 (1996).

[36] H. J. Bakker, G. C. Cho, H., Kurz Q. Wu, and X.-C. Zhang, Distortion of terahertz pulses in electro-optic sampling, J. Opt. Soc. Am. B, Vol. 15, No. 6, pp. 1795-1801 (1998).

[37] Q. Wu, and X.-C. Zhang, 7 terahertz broadband GaP electro-optic sensor, Appl. Phys. Lett., Vol. 70, No. 14, pp. 1784-1786 (1997).

[38] W. L. Faust, and C. H. Henry, Mixing of Visible and Near-Resonance Infrared Light in GaP, Phys. Rev. Lett., Vol. 17, No. 25, pp. 1265-1268 (1966).

[39] K. K. Thornber, Path Integrals and Their Applications in Quantum, Statistical and Solid-State physics, Springer, ISBN-10: 0306400170, Plenum, New York (October 1, 1978).

[40] Y. Yamashita, A. Endoh, K. Shinohara, K. Hikosaka, T. Matsui, S. Hiyamizu, and T. Mimura, Pseudomorphic $\mathrm{In}_{0.52} \mathrm{Al}_{0.48} \mathrm{As} / \mathrm{In}_{0.7} \mathrm{Ga}_{0.3} \mathrm{As}$ HEMTs with an ultrahigh fT of 562 GHz, IEEE Electron Dev. Lett., Vol. 23, No. 10, pp. 573-575 (2002).

[41] P.C.M. Planken, H.K. Nienhuys, H.J. Bakker, and T. Wenckebach, Measurement and calculation of the orientation dependence of terahertz pulse detection in $\mathrm{ZnTe}, J$. Opt. Soc. Am. B, Vol. 18, No. 3, pp. 313-317 (2001).

[42] Q. Wu, and X.-C. Zhang, Free-space electro-optics sampling of mid-infrared pulses, Appl. Phys. Lett., Vol. 71, No. 10, pp. 1285-1286 (1997).

[43] N. Sekine, and K. Hirakawa, Dispersive Terahertz Gain of a Nonclassical Oscillator: Bloch Oscillation in Semiconductor Superlattices, Phys. Rev. Lett., Vol. 94, No. 5, pp. 057408-067411 (2005).

[44] E. Gornov, E. M. Vartiainen, and K.-E. Peiponen, Comparison of subtractive KramersKronig analysis and maximum entropy model in resolving phase from finite spectral range reflectance data, Appl. Opt., Vol. 45, No. 25, pp. 6519-6524 (2006).

[45] E. M. Vartianen, K.-E., Peiponen and T. Asakura, Phase Retrieval in Optical Spectroscopy: Resolving Optical Constants from Power Spectra, Appl. Spectrosc., Vol. 50, No. 10, pp. 1283-1289 (1996).

[46] E. M. Vartiainen, Y. Ino, R. Shimano, M. Kuwata-Gonokami, Y. P. Svirko, and K.-E. Peiponen, Nemerical phase correction method for terahertz time-domain reflection spectroscopy, J. Appl. Phys., Vol. 96, pp. 4171-4175 (2004).

[47] J. B. Gunn, and C. A. Hogarth, A Novel Microwave Attenuator Using Germanium, J.Appl.Physics, Vol. 26, pp. 353-354 (1955).

[48] P. Das, and P. W. Staecker, Proc. of Sixth Int. Con. on Micr. Gen. \& Ampl., Cambridge (1966).

[49] P. J. Bulman, G. S. Hobson, and B. C. Taylor, Transferred Electron Devices, Academic Press, ISBN-10: 0121408507, London and New York (1972).

[50] P. Das, and R. Bharat, Hot electron relaxation times in two-valley semiconductors and their effect on bulk-microwave oscillators, Appl. Phys. Lett., Vol. 11, No. 12, pp. 386388 (1967). 
[51] A. F. Gibson, T. W. Granville, and E. G. S. Paige, A study of energy-loss processes in germanium at high electric fields using microwave techniques, J. Phys. Chem. Solid Vol. 19, No. 3-4, pp. 198-217 (1961).

[52] L. Reggiani, INFM - National Nano-technology Laboratory, Dipartimento di Ingegneria dell's Innovazione, Universita di Lecce. (Calculated by Professor Reggiani L.)

[53] C. Benz, and J. Freyer, $200 \mathrm{GHz}$ pulsed GaAs-IMPATT diodes, Electron. Lett., Vol. 34, No. 24, pp. 2351-2353 (1998).

[54] R. Kamoua, H. Eisele, and G. I. Haddad, D-band (110-170 GHz) InP Gunn devices DBand (110-170GHz) InP Gunn Devices, Solid-State Electron., Vol. 36, No. 11, pp. 1547-1555 (1993).

[55] M. F. Zybura, S. H. Jones, B. W. Lim, J. D. Crowley, and J. E. Carlstrom, 125-145 GHz stable depletion layer transferred electron oscillators, Solid-State Electron., Vol. 39, No. 4, pp. 547-553 (1996).

[56] J. G. Ruch, and W. Fawcett, Temperature dependence of transport properties of Gallium Arsenide determined by a Monte-carlo method, J. Appl. Phys., Vol. 41, No. 9, pp. 3843-3865 (1970).

[57] H. D. Rees, Hot electron effects at microwave frequencies in GaAs, Soli. Stat. Commun., Vol. 7, No. 2, pp. 267-270 (1969).

[58] V. Gruzinskis, E. Starikov, and P. Shiktorov, Conservation equations for hot carriers - II. Dynamic features, Solid-State Electron. Vol. 36, No. 7, pp. 1067-1075 (1993).

[59] M. V. Fischetti, Monte-carlo simulation of transport in technologically significant semiconductors of the diamond and zincblende structures. 1. Homogeneous transport, IEEE Trans. Electron Devices, Vol. 38, No. 3, pp. 634-649 (1991). 


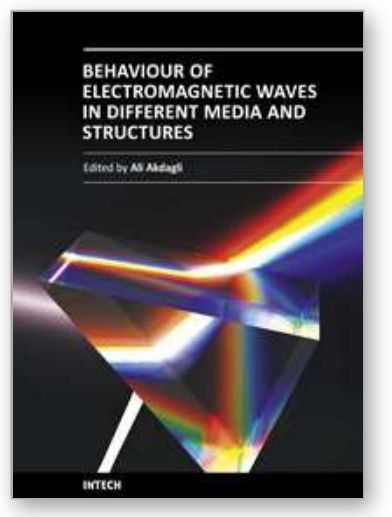

\section{Behaviour of Electromagnetic Waves in Different Media and Structures \\ Edited by Prof. Ali Akdagli}

ISBN 978-953-307-302-6

Hard cover, 440 pages

Publisher InTech

Published online 09, June, 2011

Published in print edition June, 2011

This comprehensive volume thoroughly covers wave propagation behaviors and computational techniques for electromagnetic waves in different complex media. The chapter authors describe powerful and sophisticated analytic and numerical methods to solve their specific electromagnetic problems for complex media and geometries as well. This book will be of interest to electromagnetics and microwave engineers, physicists and scientists.

\section{How to reference}

In order to correctly reference this scholarly work, feel free to copy and paste the following:

YiMing Zhu and SongLin Zhuang (2011). Ultrafast Electromagnetic Waves Emitted from Semiconductor, Behaviour of Electromagnetic Waves in Different Media and Structures, Prof. Ali Akdagli (Ed.), ISBN: 978-953307-302-6, InTech, Available from: http://www.intechopen.com/books/behavior-of-electromagnetic-waves-indifferent-media-and-structures/ultrafast-electromagnetic-waves-emitted-from-semiconductor1

\section{INTECH}

open science | open minds

\author{
InTech Europe \\ University Campus STeP Ri \\ Slavka Krautzeka 83/A \\ 51000 Rijeka, Croatia \\ Phone: +385 (51) 770447 \\ Fax: +385 (51) 686166 \\ www.intechopen.com
}

\author{
InTech China \\ Unit 405, Office Block, Hotel Equatorial Shanghai \\ No.65, Yan An Road (West), Shanghai, 200040, China \\ 中国上海市延安西路65号上海国际贵都大饭店办公楼 405 单元 \\ Phone: +86-21-62489820 \\ Fax: $+86-21-62489821$
}


(C) 2011 The Author(s). Licensee IntechOpen. This chapter is distributed under the terms of the Creative Commons Attribution-NonCommercialShareAlike-3.0 License, which permits use, distribution and reproduction for non-commercial purposes, provided the original is properly cited and derivative works building on this content are distributed under the same license. 